\title{
G192.8-1.1: A Candidate of An Evolved Thermal Composite Supernova Remnant Reignited by Nearby Massive Stars
}

\author{
Ji-HYun KAnG ${ }^{1}$, Bon-CHul Koo ${ }^{2}$, And Do-YOung BYun ${ }^{1}$ \\ ${ }^{1}$ Korea Astronomy and Space Science Institute, Daejeon 305-348, Korea; jkang@kasi.re.kr,bdy@kasi.re.kr \\ ${ }^{2}$ Department of Physics and Astronomy, Seoul National University, Seoul 151-747, Korea; koo@astro.snu.ac.kr
}

Received August 20, 2014; accepted October 22, 2014

\begin{abstract}
G192.8-1.1 has been known as one of the faintest supernova remnants (SNRs) in the Galaxy until the radio continuum of G192.8-1.1 is proved to be thermal by Gao et al. (2011). Yet, the nature of G192.8-1.1 has not been fully investigated. Here, we report the possible discovery of faint non-thermal radio continuum components with a spectral index $\alpha \sim 0.56\left(S_{\nu} \propto \nu^{-\alpha}\right)$ around G192.8-1.1, while most of the radio continuum emission is thermal. Also, our Arecibo Hi data reveal an Hi shell, expanding with an expansion velocity of $20-60 \mathrm{~km} \mathrm{~s}^{-1}$, that has an excellent morphological correlation with the radio continuum emission. The estimated physical parameters of the Hi shell and the possible association of non-thermal radio continuum emission with it suggest G192.8-1.1 to be an 0.3 Myr-old SNR. However, the presence of thermal radio continuum implies the presence of early-type stars in the same region. One possibility is that a massive star is ionizing the interior of an old SNR. If it is the case, the electron distribution assumed by the centrally-peaked surface brightness of thermal emission implies that G192.8-1.1 is a "thermal-composite" SNR, rather than a typical shell-type SNR, where the central hot gas that used to be bright in X-rays has cooled down. Therefore, we propose that G192.8-1.1 is an old evolved thermal-composite SNR showing recurring emission in the radio continuum due to a nearby massive star. The infrared image supports that the Hi shell of G192.8-1.1 is currently encountering a nearby star forming region that possibly contains an early type star(s).
\end{abstract}

Key words: shock waves — ISM: individual objects (G192.8-1.1) — ISM: supernova remnants — radio continuum: ISM — radio lines: HI

\section{INTRODUCTION}

G192.8-1.1 (PKS 0607+17, hereafter G192) has been first reported as a non-thermal source in 1969 by Milne (1969). It has been considered to be a supernova remnant (SNR) by several researchers (e.g., Caswell 1970; Day et al. 1972; Milne \& Dickel 1974; Dickel \& Denoyer 1975) due to its non-thermal radio spectral index. Berkhuijsen (1974) has also proposed that it is a part of the Origem Loop, a large, $5^{\circ}$-sized continuum shell, rather than an individual SNR. However, the high resolution $\left(\theta_{\mathrm{FWHM}} \sim 1^{\prime} .1 \times 3^{\prime} .3 \cos \delta\right)$ radio continuum observations at $1420 \mathrm{MHz}$ (Caswell 1985) revealed its $80^{\prime}$-sized circular structure as a separate SNR with a spectral index of typical shell-type SNRs, $\alpha=0.55\left(S_{\nu} \propto \nu^{-\alpha}\right)$. G192 has been regarded as one of the faintest SNRs in the Milky Way so far (Green 2009). Recently, Gao et al. (2011) found that the radio emission of G192 has a flat spectrum $(\alpha \sim 0)$ at frequencies between 1.4 and $4.8 \mathrm{GHz}$, concluding that G192 is not an SNR but a thermal source overlaid with background non-thermal point sources and an Hir region in the line of sight (LOS). The origin of the thermal radio continuum emission of G192 has not been studied yet, leaving the nature of G192 in question.

Hi 21-cm emission line observations are often carried out to identify the association of celestial objects,

CORRESPONDING AUthor: J.-H. Kang such as HiI regions, Wolf Rayet stars, or SNRs, with their environments (e.g., van der Werf \& Higgs 1990; Kothes \& Kerton 2002; Giacani \& Dubner 2004; Lee et al. 2008). The Hi emission associated with these objects, which is radiated mostly from materials sweptup by the energetic processes related to these objects, provides physical parameters, such as the energies involved, dynamical ages, and environmental conditions of the sources, which help to understand the nature of the objects and their environments. G192 has been observed in Hi 21-cm line by Koo \& Heiles (1991). They detected excess Hi emission toward G192, at velocities higher than what is allowed by the normal Galactic rotation. This requires an energetic event(s) to accelerate the gas up to such high velocities. However, the resolution of their observations with the Hat Creek telescope $\left(36^{\prime}\right)$ was not enough to determine the association between G192 and the high-velocity Hi emission. We have observed the vicinity of G192 with a good resolution $\left(3^{\prime} .6\right)$ in the Hi 21-cm hyper-fine line, using the worldlargest Arecibo $305 \mathrm{~m}$ Radio Telescope. The Arecibo data have revealed an expanding Hi shell associated with G192, which will provide physical parameters associated with the G192, as well as clues about its origin.

In this paper, we re-examine the nature of the radio continuum of G192 and report a possible detection of non-thermal radio continuum emission in the G192 area. We also report the detection of the associated HI 


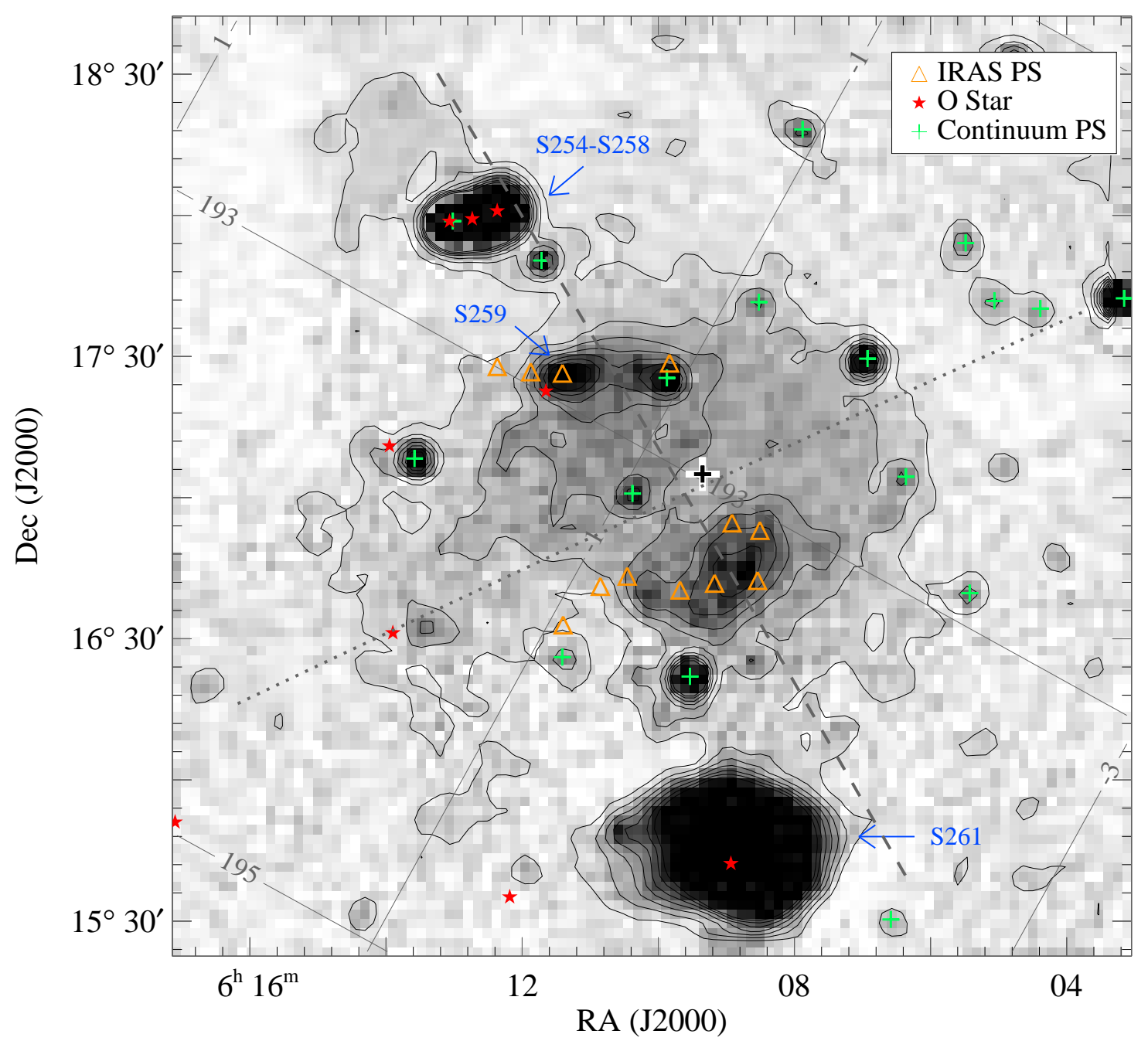

Figure 1. Effelsberg 11-cm radio continuum image of the G192 area (Fürst et al. 1990a) Contour levels start at $0.02 \mathrm{~K}$ and increase with a $0.04 \mathrm{~K}$ interval. IRAS point sources (Helou \& Walker 1988), Galactic O stars (Reed 2005), and radio continuum point sources (Fürst et al. 1990b) are presented with orange triangles, red stars, and green crosses, respectively. Sharpless HiI regions are labeled with blue colors. The dotted and the dashed lines are the lines along which 1-dimensional brightness profiles are extracted (see Figure 10).

emission in the periphery of the radio continuum structure. The new observations and the archival data used in this paper are summarized in Section 2. The results of the radio continuum analysis and the Hi observations are presented in Sections 3 and 4, respectively. In Section 5, we investigate the counterparts of G192 at multiple wavelengths, and the distance to G192. The origins of the Hi shell as well as the non-thermal and the thermal radio continuum are discussed in Section 6. We finally conclude our research in Section 7.

\section{The Radio ContinuUm Data And the Hi OBSERVATIONS}

\subsection{Radio Continuum Surveys}

For the analysis of the radio continuum emission of G192, we have used total intensity maps of the SinoGerman 6-cm (Gao et al. 2010) and the Effelsberg 21cm (Reich et al. 1997) Radio Continuum Surveys, and the NRAO VLA Sky Survey (NVSS) (Condon et al. 1998). The 6 -cm radio continuum survey data are obtained using the Urumqi $25 \mathrm{~m}$ Telescope. The data have an angular resolution of $9^{\prime} .5$ and an $\mathrm{rms}$ of $0.6 \mathrm{mK}$. The $21-\mathrm{cm}$ radio continuum survey data are obtained with the Effelesberg $100 \mathrm{~m}$ Telescope. The data have an angular resolution of $9^{\prime} .4$ and an $\mathrm{rms}$ of $40 \mathrm{mK}$. The smooth background emission is separated and released as a separate data cube. Here, we use the source data cube without the background. The released NVSS data at $21-\mathrm{cm}$ were made with a $45^{\prime \prime}$-sized restoring beam and have an rms of $140 \mathrm{mK} .^{1}$

\subsection{Hi Observations}

The observations were made during February-April, 2005 using the Arecibo $305 \mathrm{~m}$ telescope. Total inten-

\footnotetext{
${ }^{1}$ The survey data are available at http://zmtt.bao.ac.cn/6cm, http://www3.mpifr-bonn.mpg.de, and http://skyview.gsfc. nasa.gov, respectively
} 

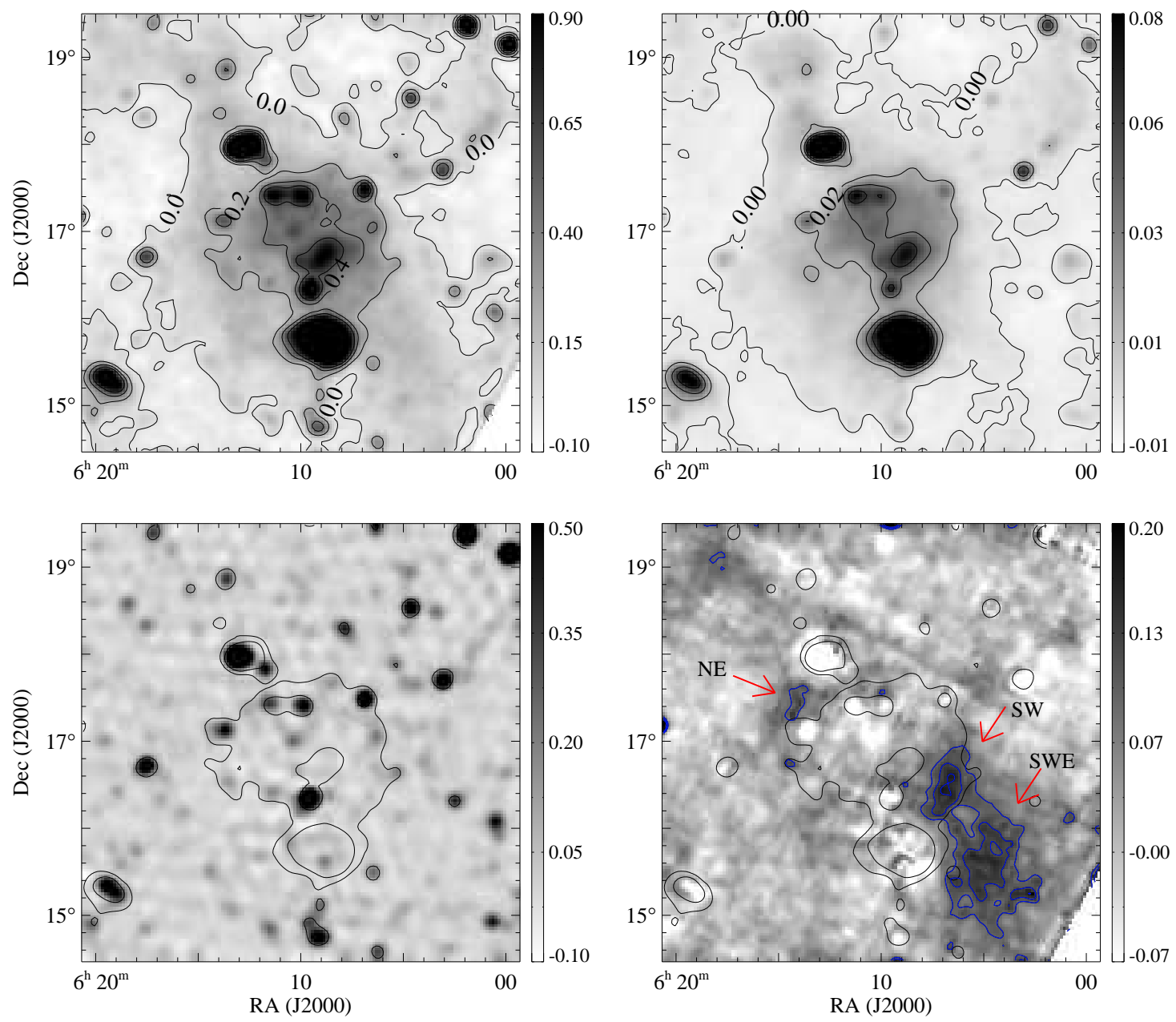

Figure 2. The Effelsberg 21-cm (top left), the Sino-German 6-cm (top right), the NVSS (bottom left) radio continuum images of the G192 field, and the non-thermal radio continuum emission, $T_{n t} f_{n t}$, (bottom right) resulting from Equation (4). The colorbars on the right of each image show the brightness temperature scales in units of $\mathrm{K}$. The 0.2 and $0.6 \mathrm{~K}$ contours of the $21-\mathrm{cm}$ image are overlaid on the images at the bottom for comparison. In the $T_{n t} f_{n t}$ image, the 3,4 , and $5 \sigma$ contours of the image, $0.09,0.12$, and $0.15 \mathrm{~K}$, are overlaid to indicate the significant excess of emission.

sity Hi 21-cm spectra were obtained with a total bandwidth of $3.125 \mathrm{MHz}$ and 1024 frequency channels using the dual-channel, L-band wide receiver. This gave a total velocity coverage of $660 \mathrm{~km} \mathrm{~s}^{-1}$, with a velocity resolution of $1.29 \mathrm{~km} \mathrm{~s}^{-1}$ after Hanning smoothing. At the same time, thermal $\mathrm{OH}$ lines at $1665 \& 1667 \mathrm{MHz}$ were obtained in a separate sub-board with the same setting as the Hi $21 \mathrm{~cm}$ lines. The area observed was of size $2.3^{\circ} \times 2.5^{\circ}$, and centered at $(\alpha, \delta) \simeq\left(6^{\mathrm{h}} 9^{\mathrm{m}}\right.$ $\left.50^{\mathrm{s}}, 17^{\circ} 00^{\prime} 00^{\prime \prime}\right)(\mathrm{J} 2000)$. Most of the area is observed using On-The-Fly (OTF) mode, scanning in $\alpha$ at a rate of $0.65 \mathrm{~s} /{ }^{\prime}$, with a half beam spacing $\left(1^{\prime} .7\right)$. However, the eastern region was scanned using OTF scans along $\delta$ at a rate of $0.48 \mathrm{~s} /{ }^{\prime}$. A single strip at $\delta=17^{\circ}$ was scanned 7 times using drift mode $\left(4 \mathrm{~s} /{ }^{\prime}\right)$ to detect any faint high-velocity Hi gas. The half-power beam width of the Arecibo telescope at this frequency is $3^{\prime} .4$.

Data were reduced using the standard IDL Arecibo data reduction software. A third-order polynomial baseline was subtracted from each spectrum using chan- nels outside the Galactic Hi line emission. The data were then gridded using a Gaussian kernal, which broadened the effective beam width to $3^{\prime} .9$. We converted the Hi spectra into brightness temperature units by multiplying it with a factor of $10.95 \mathrm{~K} \mathrm{Jy}^{-1}$, using the Leiden/Dwingeloo Hi Survey (Hartmann \& Burton $1997)$ as a reference. The mean $\mathrm{rms}(1 \sigma)$ of the final cube is $\sim 0.14 \mathrm{~K}$, while a single strip at $\delta=17^{\circ}$ has an $\mathrm{rms}$ of $0.025 \mathrm{~K}$ for $0.644 \mathrm{~km} \mathrm{~s}^{-1}$ velocity resolution.

\section{Radio Continuum Analysis}

\subsection{Radio Continuum Sources in the Fields of G192.8-1.1}

Figure. 1 shows the Effelsberg 11-cm radio continuum image (Fürst et al. 1990a) of the G192 region. The $1^{\circ} .5$ sized circular object at the center of the field is G192. A group of HiI regions, S254 - S259, are to the north, and S261 is to the south of G192. S259 is overlaid on the northeastern area of G192. The properties of these 

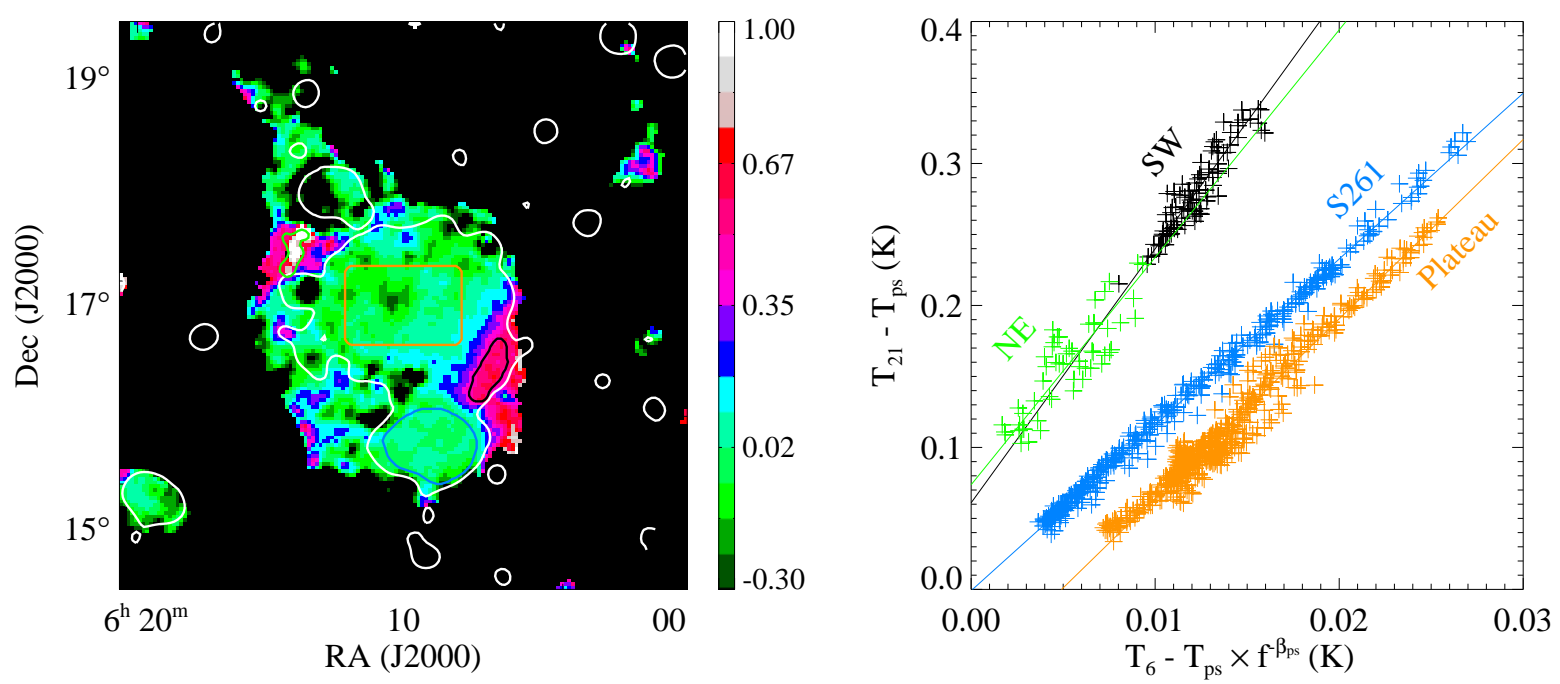

Figure 3. (Left) The spectral index $(\alpha)$ map for the pixels with $T_{21}-T_{p s}>110 \mathrm{mK}$ and $T_{6}-T_{p s} \times f^{-\beta_{p s}}>6 \mathrm{mK}$. The colorbar shows the values of $\alpha$. It it noticable that the SW and the NE areas indicated in the $T_{n t} f_{n t}$ image have non-thermal spectra. The borders of the SW, the NE, the G192 plateau, and the S261 areas, from which the spectral indices in Table 2 are estimated, are marked with black, green, orange, and blue lines, respectively. (Right) The T-T plots of the four areas indicated in the left image. Colors are the same as explained in the left image. The solid lines are the linear fits of each area. The brightness temperatures of the G192 plateau and S261 are scaled down 3 and 10 times, respectively. Also, the points in the plateau area are shifted down along the $T_{21}-T_{p s}$ axis by $0.05 \mathrm{~K}$ for better display.

HiI regions are summarized in Table 1.

The traditionally known PKS $0607+17$ SNR consists of a low-intensity plateau and the bright northern and southern rims/concentrations. The intensity of the plateau appears to generally decrease outwards. The northern rim contains S259 and point-like sources, and it is not considered to be associated with G192. The $30^{\prime}$-sized southern concentration is centered near $(\alpha, \delta) \sim\left(06^{\mathrm{h}} 09^{\mathrm{m}}, 16^{\circ} 45^{\prime}\right)$. This rim-like southern concentration was thought to be a part of G192, and G192 has been considered to be shell-type because of it.

\subsection{Radio Continuum Decomposition and the Non-thermal Component}

Gao et al. (2011) derived radio continuum spectral indices of $\alpha=0.00$ and $0.06\left(S_{\nu} \propto \nu^{-\alpha}\right)$ towards the faint plateau and the southern concentration, respectively, by fitting the temperature to temperature (T-T) plot of the plateau and the southern concentration regions using the Effelsberg 21-cm and the Sino-Germal 6-cm Radio continuum data. Their thermal spectral indices conflict with those $(\alpha \sim 0.55)$ of previous studies (Milne 1969; Caswell 1985) that are estimated at a frequency range between 178 and $2700 \mathrm{MHz}$. One explanation reconciling these two results is that the flux from the extra-galactic point sources with steeper spectrum $(\alpha \sim 1)$ affected the flux estimations of previous studies at lower frequencies. On the other hand, it is plausible that thermal and non-thermal emission co-exist in the field, resulting in a steep spectrum at lower frequencies and a flat spectrum at higher frequencies. It would be worth to investigate if the emission of G192 is all ther- mal or if there is weak non-thermal component buried within the stronger thermal emission.

For our analysis, we use the same radio continuum data sets in 21- and 6-cm as Gao et al. (2011). We can separate the thermal and non-thermal components as follows. The emission at $21-$ and $6-\mathrm{cm}$ in brightness temperature, $T_{21}$ and $T_{6}$, can be presented as

$$
\begin{aligned}
T_{21} & =T_{t h}+T_{n t}+T_{p s} \\
T_{6} & =T_{t h} f^{-\beta_{t h}}+T_{n t} f^{-\beta_{n t}}+T_{p s} f^{-\beta_{p s}}
\end{aligned}
$$

where $T_{t h}, T_{n t}$, and $T_{p s}$ are the brightness temperatures of extended thermal and non-thermal sources, and point-sources in the $21-\mathrm{cm}$ data, and $\beta_{t h}, \beta_{n t}$, and $\beta_{p s}$ represent their spectral indices. Here, $f \equiv \lambda_{21} / \lambda_{6}$ $(=21 . / 6.3=3.3)$. From the above equations, we have

$$
\begin{aligned}
T_{21-6} & \equiv T_{21}-T_{6} f^{+\beta_{t h}}=T_{n t} f_{n t}+T_{p s} f_{p s} \\
T_{n t} f_{n t} & =T_{21}-T_{6} f^{+\beta_{t h}}-T_{p s} f_{p s}
\end{aligned}
$$

where $f_{n t} \equiv\left(1-f^{-\beta_{n t}+\beta_{t h}}\right)$ and $f_{p s} \equiv(1-$

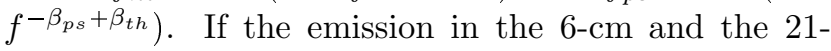
$\mathrm{cm}$ image is purely thermal, then $T_{f n} f_{n t} \sim 0$. However, if there is a non-thermal component in the data, such emission would appear as leftover emission in the $T_{f n} f_{n t}$ map, since its spectrum is steeper than that of thermal sources.

$T_{21}, T_{6}$, and $T_{p s}$ in the right-hand side of the Equation (4) can be replaced by the Effelsberg $21-\mathrm{cm}$, the Sino-German 6-cm, and the NVSS data, after the NVSS data have been convolved to a spatial resolution of $9^{\prime} .4$ and all data have been regridded to have a pixel size of $2^{\prime}$. The term related to the non-thermal component, $T_{n t} f_{n t}$, can be now determined, if $\beta_{t h}$ and $\beta_{p s}$ 

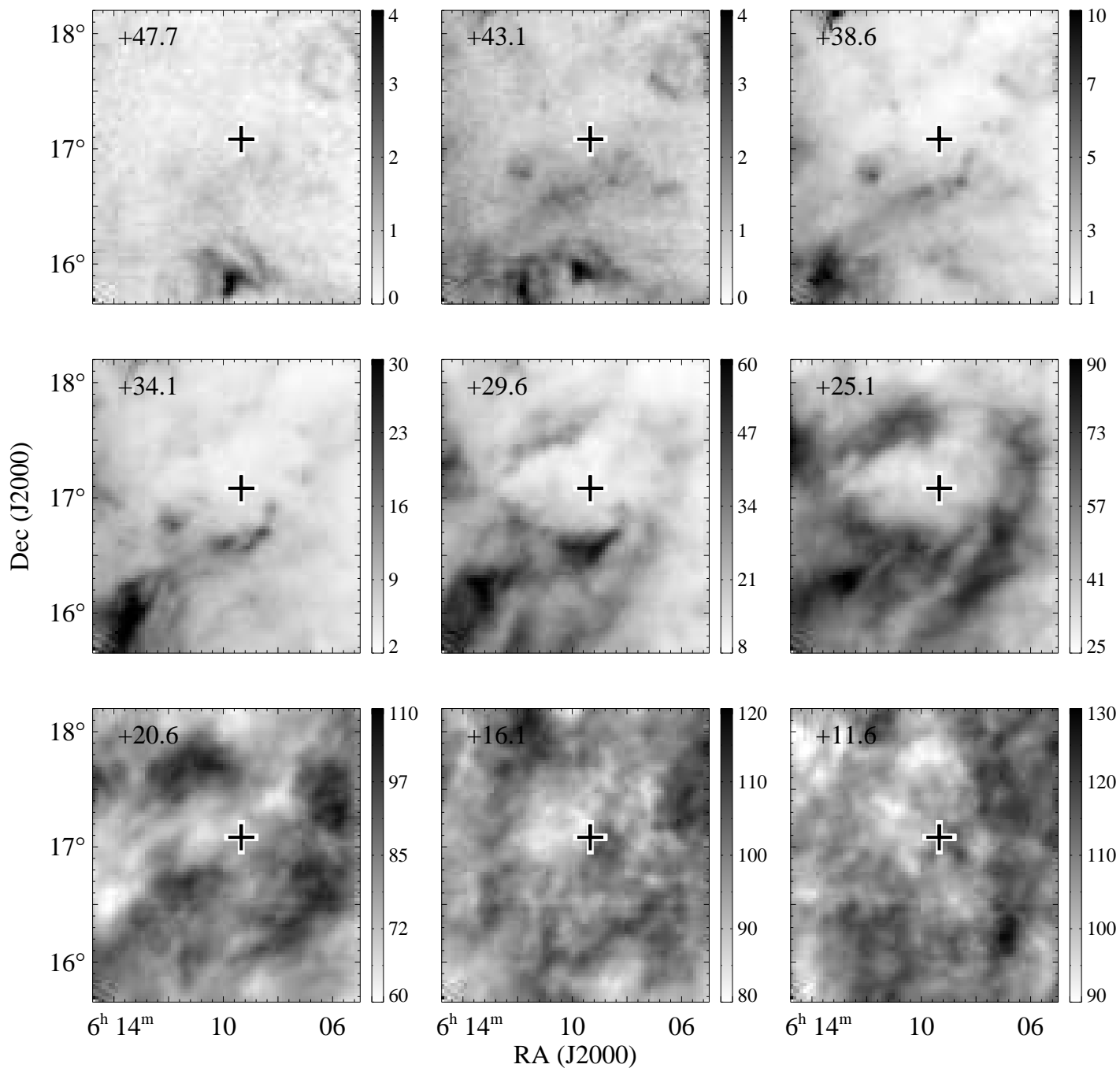

Figure 4. Hi channel images of the observed field. Each image is averaged over a velocity interval of $4.5 \mathrm{~km} \mathrm{~s}^{-1}$. The central LSR velocity is given at the top left of each image. The colorbars present the brightness temperature scales of individual channel images in Kelvin. The black cross indicates the approximate center of the radio continuum emission for G192.

are adopted. We adopt $\beta_{p s}=2.91 \pm 0.22$, which is the mean of the spectral indices of the point-sources in the G192 field given by Caswell (1985), and $\beta_{t h}=2.06$, which is based on the spectral index map that will be described in the following paragraphs. We note that $\beta_{t h}$ in the range of $2.0<\beta_{t h}<2.1$ does not change the results described in the following paragraph. Also, we note that the spectral index variation of the extragalactic sources can be larger, but it would not be critical for the detection of extended non-thermal components.

Figure 2 shows the image of resulting non-thermal component term of $T_{n t} f_{n t}$ as well as those substituted in the $T_{21}, T_{6}$, and $T_{p s}$ terms. In the $T_{n t} f_{n t}$ image (bottom right), we can notice that the G192 structures, i.e., the plateau and the southern concentration, as well as the HII regions and the diffuse continuum emission around G192 are mostly removed, confirming their thermal nature. The emission of the NVSS point sources with $\alpha<0.91$ and the HII regions with small diameters detected in the NVSS data are removed more than they should be and appear as negative holes in $T_{n t} f_{n t}$ map, while the NVSS sources with $\alpha>0.91$ survive in this map. We also notice that there is some emission left that exceeds $3 \sigma$ level $(0.09 \mathrm{~K})$ along the boundaries of G192 and beyond toward the southwest in the $T_{n t} f_{n t}$ map. The non-thermal component in the northeast (NE) is located outside the border of the $0.2 \mathrm{~K}$ contour, while the one in the southwest (SW) is rather consistant to the boundary of $0.2 \mathrm{~K}$ contour. Also, there is emission excess extended in southwest direction (SWE) outside the $0.2 \mathrm{~K}$ contour. The excess emission at the southwestern boundary of G192, SW, could be associated with the more extended emission, SWE, however, the SW appears brighter along the boundary of G192, which might indicate its association with G192.

The thermal and non-thermal nature of the sources in this field can be clarified in the pixel-to-pixel spectral index $(\alpha)$ map shown in Figure 3, which is defined as $\left(T_{21}-T_{p s}\right) /\left(T_{6}-T_{p s} f^{-\beta p s}\right)=f^{-(\alpha+2)}$. In 

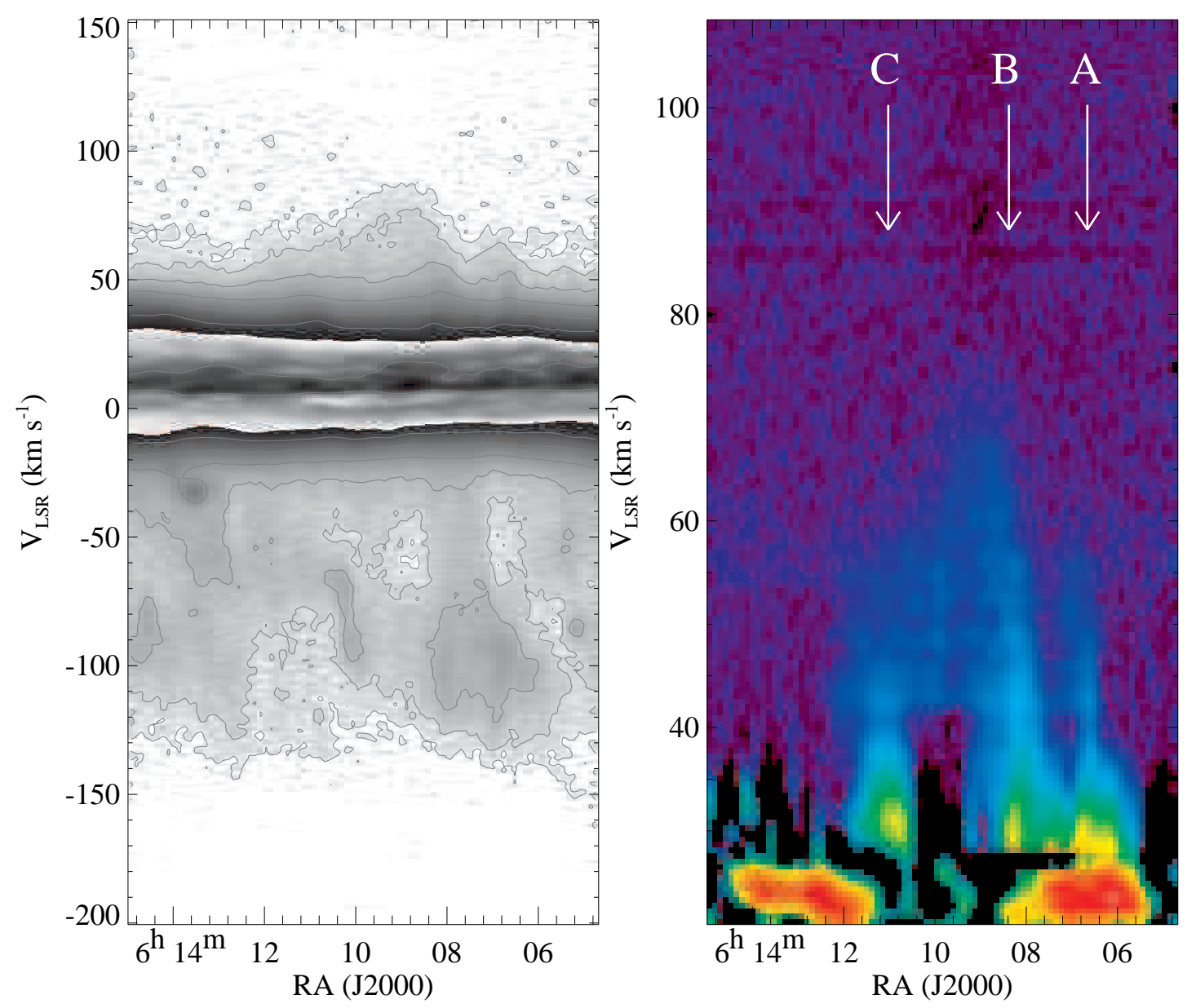

Figure 5. (Left) The sensitive $\left(v_{\mathrm{LSR}}-\alpha\right)$ slice of the Hi data at $\delta=17^{\circ}$ with contours of $0.03,0.1,0.3,1,3,10,30$, and $100 \mathrm{~K}$ in brightness temperature. The color is scaled logarithmically for temperatures $\mathrm{T}_{b}<30$, and linearly for $\mathrm{T}_{b} \geqslant 30 \mathrm{~K}$, to highlight the low- and high-intensity Hi features at the same time. (Right) Same as the one in the left zoomed at $+40<v_{\mathrm{LSR}}<+90 \mathrm{~km} \mathrm{~s}^{-1}$. The smooth background emission is removed to bring up the faint shell structure. The color is scaled logarithmically. Three wings mentioned in the text are labeled as A, B, and C.

this spectral index map, the thermal and non-thermal regions are distinguishable with green and red colors, and the regions with emission excess in Figure 2 show non-thermal spectra. We have estimated the spectral indices of four areas, i.e., the SW and the NE regions, the plateau, and the S261 regions, of which boundaries are indicated with black, green, yellow, and blue solid lines, respectively, in Figure 3 (left). The spectral indices measured from total fluxes using images of $T_{21}-T_{p s}$ and $T_{6}-T_{p s} f^{-\beta p s}$, and the medians of the pixel-to-pixel spectral index map are presented in Table 2 . We have also estimated the spectral indices of the same areas using the T-T plot method, as shown in Figure 3 (right), and the results are presented in the same table. The plateau and S261 consistently show thermal spectra of $\alpha=0.04-0.11$, independent of the method used. The SW region also shows consistant numbers of $\alpha=0.40-0.57$ with a reasonable error range of 0.15 . In contract, the spectral index $\alpha=0.31 \pm 0.23$ of the $\mathrm{NE}$ region esimated from the T-T plot method is flatter than those $(\alpha \sim 0.84 \pm 0.35)$ of other methods. The dif- ference could come from the background removed from the image. We have removed constant values of $0.047 \mathrm{~K}$ and $-0.011 \mathrm{~K}$ from the $T_{21}-T_{p s}$ and the $T_{6}-T_{p s} f^{-\beta p s}$ images, respectively. The removed constants will not affect on the slope in the T-T plot, but can be critical for the $\alpha$ values of faint regions, such as the NE region, in the pixel-to-pixel $\alpha$ map. The removed constants do not appear to affect much the results of brighter regions including the SW region.

In summary, there are some non-thermal areas, and the one extended along the southwest boundary of the G192 plateau, SW, could be associated with the G192 structure, though not conclusively. Most of the sources in the G192 field, including the plateau and the southern concentration, are confirmed to be thermal.

\section{Hi Results}

\subsection{Hi Morphologies}

We have detected an expanding shell around G192. Figure 4 shows channel images of the Arecibo Hi data. The Hi emission around the center of G192 appears 

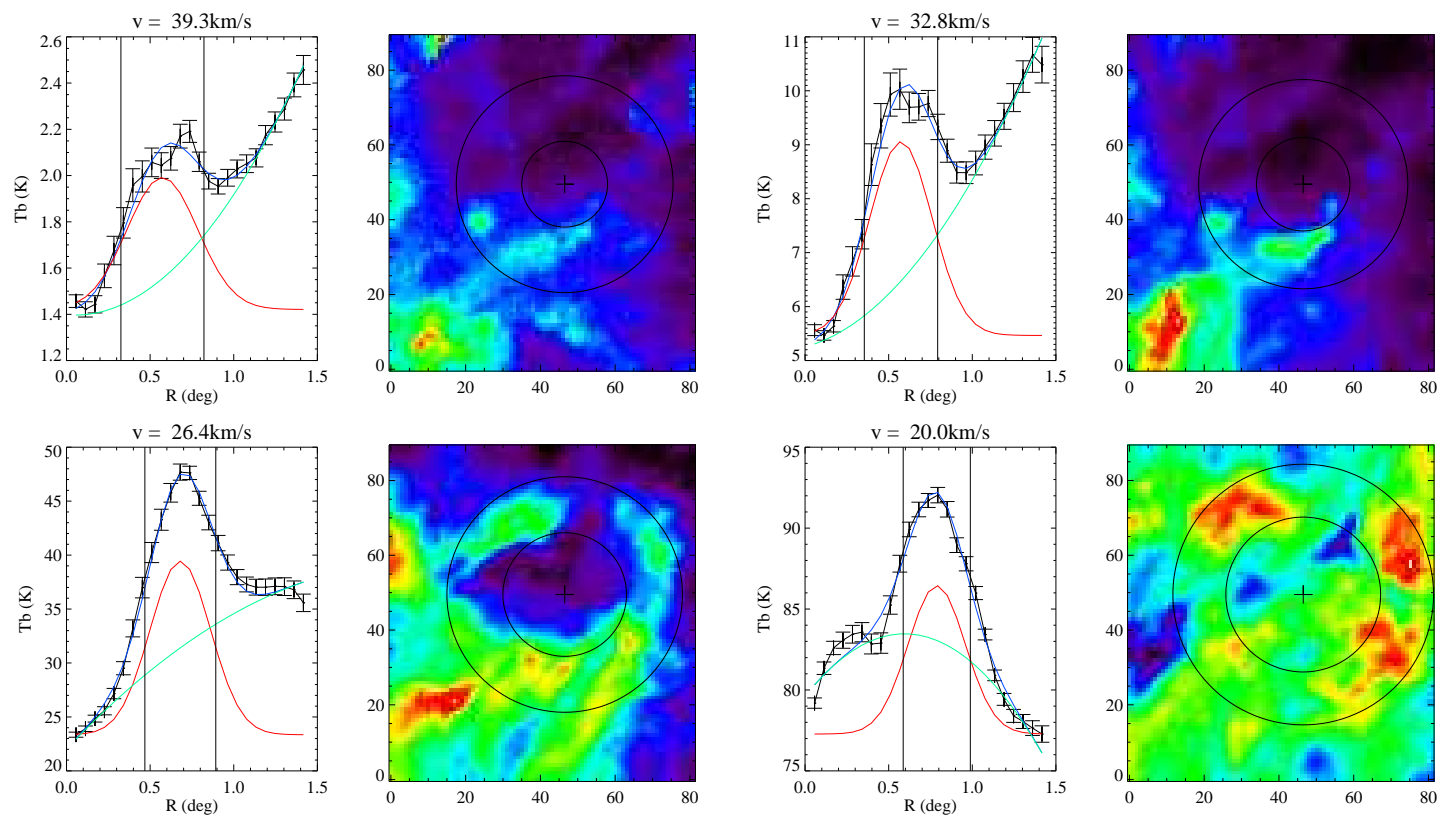

Figure 6. Examples of radial profiles and images of the Hi shell are presented at four velocity channels, which are noted in each panel. The fitted Gaussian, polynomial, and the summation of both components are shown in red, green, and blue solid lines, respectively. The resulting FWHMs of the Gaussian components presented with the two vertical solid lines in the radial profiles are also overplotted on the corresponding channel images with annulii. The Gaussian components appear to represent the shell component well.

as ring-like structures at $v_{\mathrm{LSR}}=+20.6,+25.1$, and $+29.6 \mathrm{~km} \mathrm{~s}^{-1}$. The ring structure is most prominent at $v_{\mathrm{LSR}}=+25.1 \mathrm{~km} \mathrm{~s}^{-1}$ channel image. The northeastern shell at $v_{\mathrm{LSR}}=+29.6 \mathrm{~km} \mathrm{~s}^{-1}$ shows a rimbrightened feature. The emission associated with the shell, while weak, can be detected along the boundary of the shell at $v_{\mathrm{LSR}}=+16.1 \mathrm{~km} \mathrm{~s}^{-1}$, but it disappears at lower velocities. In the images at higher velocities, $+34.1 \lesssim v_{\text {LSR }} \lesssim+47.7 \mathrm{~km} \mathrm{~s}^{-1}$, the Hi emission associated with the shell is weak, but visible. The HI emission at these velocities consists of a few relatively bright spots rather than a ring-like structure. In the south/southeastern areas of the observed field, there are other high velocity HI emission outside the shell boundary at $+29.6 \lesssim v_{\mathrm{LSR}} \lesssim+47.7 \mathrm{~km} \mathrm{~s}^{-1}$. The HI clouds at $v_{\text {LSR }}=+47.7 \mathrm{~km} \mathrm{~s}^{-1}$ show interesting velocity structures, but they do not appear to be associated with the shell. The Hi feature in the northwest corner of the image at $v_{\mathrm{LSR}}=+47.7 \mathrm{~km} \mathrm{~s}^{-1}$ is FVW191.9-1.5, which is suspected to be an expanding shell of an old SNR located in the Outer Arm (Kang \& Koo 2007).

The sensitive $(v-\alpha)$ slice observed at $\delta=17^{\circ}$ with seven drift scans (Section 2) reveals fainter high velocity structure of the expanding shell. Figure 5 (left) shows that Hi excess exists well above $+50 \mathrm{~km} \mathrm{~s}^{-1}$ up to $\sim+90 \mathrm{~km} \mathrm{~s}^{-1}$. Note that the maximum velocity allowed in this direction is $v_{\mathrm{LSR}} \sim 30 \mathrm{~km} \mathrm{~s}^{-1}$, when a flat rotation velocity of $220 \mathrm{~km} \mathrm{~s}^{-1}$ and the distance to the Galactic Center of $8.5 \mathrm{kpc}$ are adopted. A fit of a Gaussian plus 1-degree polynomial to areas not associated with the shell is removed from the intensity profile of the $(v-\alpha)$ slice at each velocity channel as a background to emphasize the shell structure. The result is presented in the right panel of Figure 5. The expansion of the shell is prominently visible in this background removed image. There are three faint wings at $v_{\text {LSR }} \gtrsim+30 \mathrm{~km}$ $\mathrm{s}^{-1}$. The wings look slightly curved inward. The two wings near $\alpha \sim 6^{\mathrm{h}} 8^{\mathrm{m}}$ (B in the figure) and $\sim 6^{\mathrm{h}} 11^{\mathrm{m}}(\mathrm{C})$ are connected and show a loop-like structure at velocities above $\sim+30 \mathrm{~km} \mathrm{~s}^{-1}$ in the $(v-\alpha)$ slice, implying its expansion. It looks like that the receding endcap of the shell is located at velocities between +50 and $+70 \mathrm{~km} \mathrm{~s}^{-1}$ toward this direction. The expansion velocity implied from this slice is $40-60 \mathrm{~km} \mathrm{~s}^{-1}$, when adopting a systemic velocity of $v_{\mathrm{LSR}}=+10 \mathrm{~km} \mathrm{~s}^{-1}$, the systemic LSR velocity of the S254-259. The emission of $\mathrm{B}$ and $\mathrm{C}$ peaks at $v_{\mathrm{LSR}}=+30-+35 \mathrm{~km} \mathrm{~s}^{-1}$, and looks disconnected towards lower LSR velocities. The wall near $\alpha \sim 6^{\mathrm{h}} 6^{\mathrm{m}} .5$ (A) appears thick and bright at $+20 \lesssim v_{\text {LSR }} \lesssim+30 \mathrm{~km} \mathrm{~s}^{-1}$, and it appears gradually thinner and fainter at higher velocities. The sensitive $(v-\alpha)$ slice also shows high velocity Hi gas at negative forbidden velocities. Negative forbidden velocities are confusing mainly due to the large Anti-Center Shell (ACS; Tamanaha 1997). The approaching part of the Hi shell may not be visible because of this confusion.

\subsection{Properties of the Hi Shell}

We have measured the Hi intensity as a function of radius by averaging pixels within a same annulii at every $0.64 \mathrm{~km} \mathrm{~s}^{-1}$ velocity channel in the velocity range of $v_{\text {LSR }}=+19.3$ and $+42.5 \mathrm{~km} \mathrm{~s}^{-1}$. The emission from the shell appears as a bump in the example radial profiles in Figure 6. We fit the radial profiles with a function of 

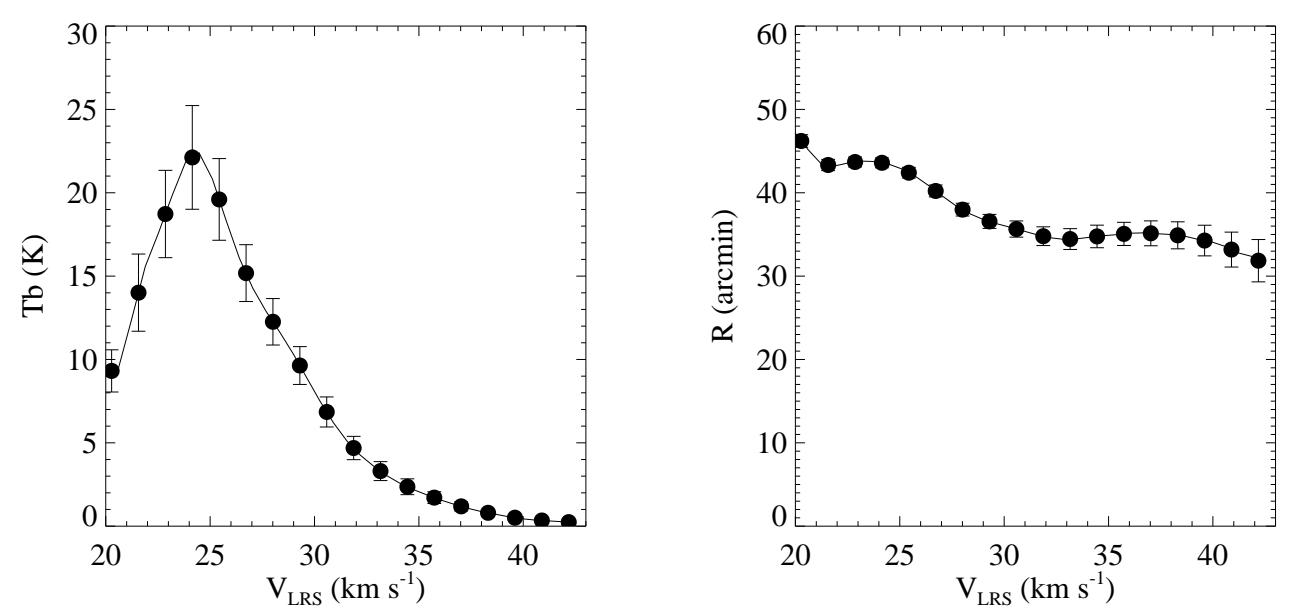

Figure 7. The mean brightness temperature and the radius of the shell as a function of velocity, which result from the Gaussian fit of the radial profile. The shown error is $3 \sigma$.

a Gaussian plus 2nd degree polynomial, i.e., emission from the shell and the background. These components are shown with red and green lines in the profiles. The positions of the full width at half maximum (FWHM) of the resulting Gaussian components are overlaid on the channel images. As shown in the radial profiles and the channel images, the Gaussian fit appears to be a reliable method in tracing the Hi shell.

The mean brightness and the radius of the shell resulting from the Gaussian fit are presented in Figure 7 as a function of the LSR velocity. The shell is most bright $\left(\bar{T}_{b} \sim 23 \mathrm{~K}\right)$ around $v_{\mathrm{LSR}} \sim 24 \mathrm{~km}$ $\mathrm{s}^{-1}$ in average, while the radius tends to decrease as the LSR velocity increases. The maximum mean radius is $46^{\prime}$ at $+20 \mathrm{~km} \mathrm{~s}^{-1}$, and it decreases down to $32^{\prime}$ at $+42 \mathrm{~km} \mathrm{~s}^{-1}$, which is evidence of expansion. The change of the radius along the velocity does not exactly follow the ideal ellipse with a relation of $\left(r-r_{0}\right)^{2}+\left(v_{\text {sys }}-v_{\text {exp }}\right)^{2}=1$, which would result in a slow change in radius near the systemic velocity (lower velocity) and rapid change near the endcap velocity (higher velocity). Rather it has some variations in the curvature of radius. This variation may be influenced by which part of the shell dominates the Gaussian component or how well the background is removed. For example, at higher velocities, the shell component is only constrained through the shell emission in the south, but at lower velocities, the shell is relatively well defined in a circle in all directions. The FWHM of the northern portion of the shell at $v_{\mathrm{LSR}}=25.1 \mathrm{~km} \mathrm{~s}^{-1}$ is $20^{\prime}$, which is more than $40 \%$ of the largest radius measured, implying that the shell is significantly thick.

The HI mass estimated from the Gaussian component at $+19.9 \leqslant v_{\mathrm{LSR}} \leqslant+42.5 \mathrm{~km} \mathrm{~s}^{-1}$ is $1300 \mathrm{~d}_{\mathrm{kpc}}^{2}$ $\mathrm{M}_{\odot}$ with an uncertainty of $10 \%$, where $\mathrm{d}_{\mathrm{kpc}}$ is the distance in units of kpc. The estimated mass is similar to the one estimated from the integrated image within the error. There is HI gas at high velocities according to our sensitive $(p-v)$ map, but it is not visible in the low-sensitivity map. The mean hydrogen column density of the high velocity gas in the 1-dimensional map integrated at $+42.5 \leqslant v_{\text {LSR }} \leqslant+90 \mathrm{~km} \mathrm{~s}^{-1}$ is $\mathrm{N}_{\mathrm{HI}}=2.4 \times 10^{18} \mathrm{~cm}^{-2}$. Assuming that this is general value for the entire receding portion of the shell, the estimated mass at $v_{\mathrm{LSR}} \geqslant+42.5 \mathrm{~km} \mathrm{~s}^{-1}$ is $\sim 10 \mathrm{~d}_{\mathrm{kpc}}^{2} \mathrm{M}_{\odot}$ , which is negligible in comparison to the mass estimated at lower velocities. The HI mass distribution along the LSR velocity is shown in Figure 8. The mass at $+19.3 \leqslant v_{\text {LSR }} \leqslant+29.0 \mathrm{~km} \mathrm{~s}^{-1}$ occupies $85 \%$ of the observed Hi mass, meaning that the observed mass is mainly determined by the bright shell at these velocities. The mass distribution decreases at $v \lesssim+24 \mathrm{~km}$ $\mathrm{s}^{-1}$. The mass at these velocities could be underestimated due to the confusion from Hi self-absorption and strong fore- and background emission. Alternatively it could be due to the inhomogeneity of the shell. We discuss the total mass of the Hi shell in Section 6.1.1.

\section{MUlti-WaVelength VieW OF G192.8-1.1 AND THE DisTaNCE}

\subsection{Radio Continuum and the HI $21-\mathrm{cm}$ Line}

There is a good spatial correlation between the HI and the radio continuum of G192, implying their association. In Figure 9, the blue shell at $v_{\mathrm{LSR}}=+20-$ $+25 \mathrm{~km} \mathrm{~s}^{-1}$ is well defined along the radio continuum boundary of $\mathrm{G} 192, \mathrm{~T}_{b}=0.06 \mathrm{~K}$, where the centrally brightened radio continuum emission starts to decrease outward (see also Figure 1$)$. The Hi in red $\left(v_{\mathrm{LSR}}=+30\right.$ $\left.-+40 \mathrm{~km} \mathrm{~s}^{-1}\right)$ is also visible in two spots along the inner boundary of the blue and green shell. In particular, there is a tight spatial correlation between the Hi and the southern radio continuum concentration. We note that the emission at higher velocities in green and red colors outside the shell feature to the southeast, which is also visible in the channel images, does not appear to be associated with the Hi shell.

G192 is known as a shell-type SNR because of the 


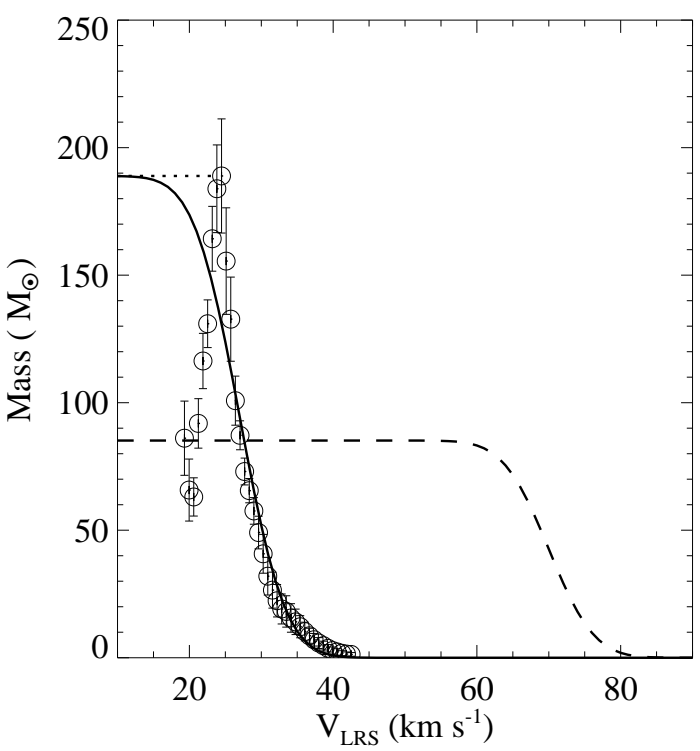

Figure 8. Hi mass per $1 \mathrm{~km} \mathrm{~s}^{-1}$ velocity interval normalized to $1 \mathrm{kpc}$. The observed Hi mass is shown in open circles with $1 \sigma$ error. The adopted extrapolation of mass near the central velocity is shown with the flat short-dashed line. The solid and long-dashed lines are the expected mass distributions for a shell with $v_{\exp }=17$ and $60 \mathrm{~km} \mathrm{~s}^{-1}$, respectively, when the velocity dispersion of the swept-up material is $5 \mathrm{~km} \mathrm{~s}^{-1}$ and the shell is assumed to be spherically symmetric.

bright concentration in the south and possibly the extended emission in the north connecting S259 and its nearby point sources. Other than this, the continuum emission is not particularly bright along the edge (Figure 1). Instead, the intensity of the radio continuum decreases where the Hi shell is strong, except for the southern continuum concentration region. This trend is more apparent in Figure 10, which compares intensity profiles of the Effelsberg $11-\mathrm{cm}$ radio continuum and the HI across the lines shown in Figure 1. The right panel in Figure 10 shows that the radio continuum (red) is brighter in the center and does not have particular rim-brightened features, but the Hi peaks (black) are well defined where the radio continuum becomes fainter. This is general features in G192. For the slice where the radio continuum peaks are visible (left in Figure 10), the continuum peaks are closer to the center than the Hi peaks. The positional shifts of the radio continuum to the Hi shown in these figures can be interpreted as the continuum being emitted from the inner area of the Hi shell. The flat brightness profile of the radio continuum is either filling the entire volume inside the shell or, if it is a shell, the shell must be thick.

The radio continuum emission mentioned above is mostly thermal. The non-thermal radio continuum emission is compared with the HI shell at $v_{\mathrm{LSR}}=$ $+21 \mathrm{~km} \mathrm{~s}^{-1}$ in Figure 11. The SW emission is well correlated with the position of the Hi shell, while the NE and SWE non-thermal emission do not appear to be associated with the Hi shell.

\subsection{Infrared Emission}

Figure 12 shows the RGB composite image of the Widefield Infrared Survey Explorer (WISE) data at 4.5, 12, and $22 \mu \mathrm{m}$, with contours of the Effelsberg $11-\mathrm{cm}$ radio continuum overlaid. The radio continuum plateau indicated with the $0.06 \mathrm{~K}$ contour does not have a counterpart in the infrared, while the northern and the southern radio continuum rims traced with the $0.18 \mathrm{~K}$ contour have corresponding infrared emission. The northern infrared counterparts are probably located in the Outer Arm, where S259 resides. It does not show apparent correlation with the Hi shell.

The association between the southern continuum rim and the Infrared Astronomical Satellite (IRAS) data (Neugebauer et al. 1984) has been reported in earlier studies (Saken et al. 1992; Arendt 1989). The high resolution WISE image presents their association in detail. The WISE counterpart of the southern continuum rim appears as an U-shaped infrared bubble-like feature opened to the north. The southern continuum peak is located in the eastern area of this infrared structure. The infrared filaments bound this region to the east and the south and show very good correlations with the compressed feature of the HI shell in Figure 13. The eastern area of this Ushaped region shows structures different from the infrared filaments associated with the Hi shell to the west. There are several infrared cores (point sources) with tail-like features spread outward. The brightest square-shaped infrared source, IRAS $06075+1643$, at $(\alpha, \delta) \sim\left(6^{\mathrm{h}} 10^{\mathrm{m}} 27^{\mathrm{s}} .4,+16^{\circ} 43^{\prime} 18^{\prime \prime}\right)$, which contains multiple infrared point sources, also shows a compressed feature inward with a tail spread outward. Those headtail infrared structures are seen in star forming regions, such as the "horse-head nebula", where photons from massive stars ionize nearby molecules and reveal embedded young stellar objects. The directions of these head-tail features indicate that the central star is located somewhere near $(\alpha, \delta) \sim\left(6^{\mathrm{h}} 09^{\mathrm{m}} 40^{\mathrm{s}},+16^{\circ} 52^{\prime}\right)$. Nevertheless, there is no obvious central star responsible for the feature (see also Section 6.2).

\subsection{Distance to G192.8-1.1}

It is very probable that G192 is associated with the HII regions in the LOS, since early type stars, the likely progenitors of expanding Hi shells in the Galactic Plane, tend to be born in groups in molecular clouds. Here, we investigate the distances of G192 based on the distances of the 7 HiI regions shown in Figure. 1, which are summarized in Table 1.

The S254 - S258 Hin regions are known to be associated with the Gemini OB1 molecular cloud complex (Carpenter et al. 1995), which make up the Perseus Arm. The spectro-photometric distance to the stars embedded in these HII region complex is derived separately by Russeil et al. (2007) and Moffat et al. (1979) give a distance of $2.5 \mathrm{kpc}$. However, the recently measured trigonometric parallax to the $6.7 \mathrm{GHz}$ methanol 


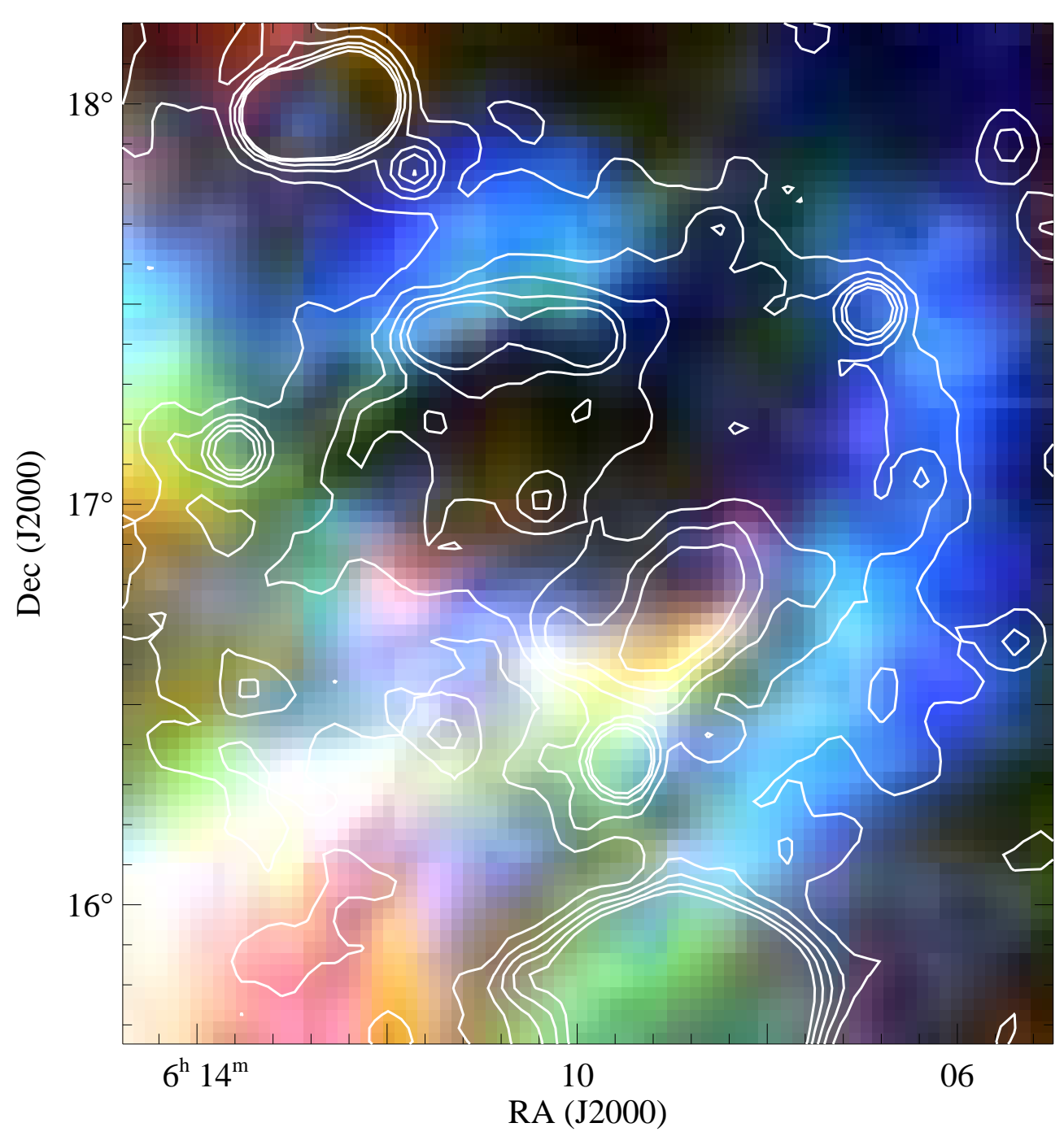

Figure 9. The HI images averaged over $v_{\mathrm{LSR}}=+20-+25,+25-+30$, and $+30-+40 \mathrm{~km} \mathrm{~s}^{-1}$ are presented in blue, green, and red, respectively. White contours are the Effelsberg $11-\mathrm{cm}$ radio continuum emission at $0.02,0.06,0.1,0.14$, and $0.18 \mathrm{~K}$ in brightness temperature. See also Figure 1.

maser of S255 gives a closer distance, $1.6 \mathrm{kpc}$ (Rygl et al. 2010), implying that the Gemini OB1 molecular cloud is the this distance. The spectroscopic or photometric distances to the star within S261 are $1.4 \mathrm{kpc}$ (Georgelin \& Georgelin 1976) and $1.5 \mathrm{kpc}$ (Garmany et al. 1982), which are close to that of S255. The radial velocity of the $\mathrm{H} \alpha$ line emission of S261, $v_{\mathrm{LSR}}=+5.6 \mathrm{~km}$ $\mathrm{s}^{-1}$ (Hausen et al. 2002) is close to the lower limit of those of S254-S258, $v_{\mathrm{LSR}}=+6.0-+11.1 \mathrm{~km} \mathrm{~s}^{-1}$, measured in $\mathrm{CO}$ and $\mathrm{H} \alpha$ lines. On the other hand, S259, which overlaps with G192, seems separated from the rest of the HII regions in the field. The spectroscopic distance to its central star put S259 at a distance of 8.3 $\mathrm{kpc}$ (Moffat et al. 1979). The radial velocities measured from $\mathrm{CO}$ and $\mathrm{H} \alpha$ lines, $v_{\mathrm{LSR}}=+22.8$ and $+15.8 \mathrm{~km} \mathrm{~s}^{-1}$ (Blitz et al. 1982; Fich et al. 1990), also support it being at a larger distance. In summary, the HiI regions in the LOS of G192, seem to be at two different places, a group in the Perseus Arm $(\sim 1.5 \mathrm{kpc})$ and the other in the Outer Arm $(\sim 8.3 \mathrm{kpc})$. We adopt a closer distance of $1.5 \mathrm{kpc}$ as a distance to G192, but its properties at a far distance will be discussed, too.

\section{ORIGIN OF G192.8-1.1}

The non-thermal radio continuum emission appears to be correlated with the Hi shell, while the thermal radio continuum component seems to be confined within the Hi shell. The expanding Hi shell with associated nonthermal emission is a typical feature of an evolved SNR. On the other hand, the thermal radio continuum surrounded by an expanding Hi shell is a common feature for an His region or stellar wind bubbles. To understand the nature of G192, we investigate the properties of the Hi shell and the thermal radio continuum emission, and discuss possible explanations of the G192 complex as a whole. 

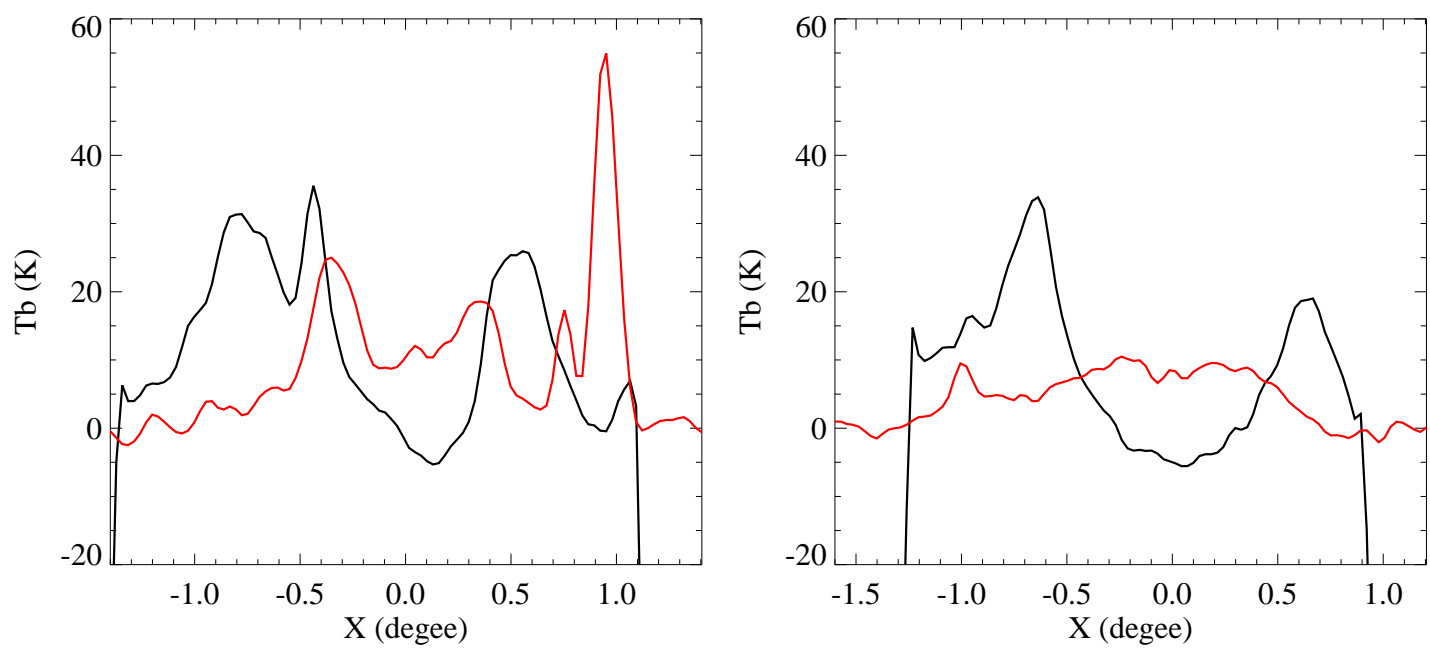

Figure 10. Intensity profiles of the radio continuum at $11-\mathrm{cm}$ (red) and the HI (black) averaged over $+20 \leqslant v_{\mathrm{LSR}} \leqslant+50 \mathrm{~km}$ $\mathrm{s}^{-1}$ along the dashed (left) and the dotted (right) lines shown in Figure 1. The unit of radio continuum is scaled by a factor of 100 for visualization.

\subsection{Hi Shell}

\subsubsection{Hi Mass Extrapolation}

If the Hi shell is uniform and expanding at a constant speed $v_{\text {exp }}$, the average Hi profile would appear as a square profile that extends from $-v_{\text {exp }}$ to $+v_{\text {exp }}$ centered at the systemic velocity $v_{0}$. When the velocity dispersion is considered, the observed Hi profile would have a shape of a flat profile convolved with a Gaussian profile along the velocities (e.g., Park et al. 2013; Kang et al. 2012). The Hi mass follows the same shape of the HI profile along the velocities. If we define $M(v)$ as the Hi mass measured at $v$ in a velocity interval of $\Delta v=1 \mathrm{~km} \mathrm{~s}^{-1}$ and the dispersion velocity of random motion is $v_{\sigma}$, the expected mass distribution would be

$$
M(v) \propto \int_{-\infty}^{\infty} M_{\text {square }}(u) \exp \left[-\frac{(v-u)^{2}}{v_{\sigma}^{2}}\right] d u
$$

where $M_{\text {square }}(v)=M_{\max }$ in $\left|v-v_{\text {sys }}\right|<v_{\text {exp }}$ and zero otherwise. Two examples of this profile with different $v_{\text {exp }}$ and $M_{\max }$ are shown in Figure 8 . In addition to this, when the shell is integrated over a certain velocity width, the mass of the inner region of the shell at a channel will be underestimated in the process of removing backgrounds, if the bubble with extremely low density is included in the integrated velocity range. This effect will produce a dip in the observed mass profile at velocities near $v_{\text {sys }}$ (cf., Cazzolato \& Pineault 2005). The height and width of the dip depend on the thickness of the shell and the velocity dispersion of the ambient medium. The observed decrement of mass at velocities from $v_{\mathrm{LSR}} \sim 24 \mathrm{~km} \mathrm{~s}^{-1}$ down to $v_{\mathrm{LSR}} \sim 20 \mathrm{~km} \mathrm{~s}^{-1}$ in Figure 8 could be partially affected by this effects.

In Figure 8, it is obvious that the observed mass distribution does not fit with the profile of the isotropic shell with uniform expansion velocity of $60 \mathrm{~km} \mathrm{~s}^{-1}$ (dashed line). Rather it better fits to that of $v_{\exp }$ $\sim 20 \mathrm{~km} \mathrm{~s}^{-1}$ (solid line). This indicates that the partial shell observed at $+20 \lesssim v_{\mathrm{LSR}} \lesssim+40 \mathrm{~km} \mathrm{~s}^{-1}$ is massive, while the swept-up mass of the end part of the receding shell with $v_{\exp }=+60 \mathrm{~km} \mathrm{~s}^{-1}$ is negligibly small.

We simply assume that the mass in between the velocities with the peak mass value is constant with the maximum observed mass (dotted line connecting the mass peaks). We further assume that the mass distribution is symmetric with respect to the $v_{\text {sys }}$. This is a reasonable assumption since the energy injected by a central progenitor would be symmetric, even if the surrounding medium is anisotropic. The systemic velocity of G192 is not known, since the shell is only partially detected. Here, we adopt $v_{\text {sys }}=+10 \pm 5 \mathrm{~km} \mathrm{~s}^{-1}$, which is close to the systemic velocities of S261 or S254 $\mathrm{S} 258\left(v_{\mathrm{sys}}=5.6-11.1 \mathrm{~km} \mathrm{~s}^{-1}\right)$. Note that $v_{\text {sys }}$ towards $\ell=192^{\circ}$ varies in a relatively narrow range of $v_{\text {LSR }} \sim 0$ to $+20 \mathrm{~km} \mathrm{~s}^{-1}$ in a large distance range of $d=0-10 \mathrm{kpc}$ (Brand \& Blitz 1993). The total HI mass at a distance of $1.5 \mathrm{kpc}$ and at $v_{\mathrm{sys}}=+10 \mathrm{~km}$ $\mathrm{s}^{-1}$ is $1.5 \times 10^{4} \mathrm{M} \odot$ with $30 \%$ of mass variation in $v_{\text {sys }}$ $=+10 \pm 5 \mathrm{~km} \mathrm{~s}^{-1}$.

\subsubsection{Energetics}

As shown in Figure 8, the mass profile of the Hi shell better fits to that of a shell with $v_{\exp } \sim 20 \mathrm{~km} \mathrm{~s}^{-1}$, though the expansion velocity of the shell can be larger than this as shown in Figure 7 (right). Here, we adopt $v_{\text {exp }}=20 \mathrm{~km} \mathrm{~s}^{-1}$ as a characteristic expansion velocity of the Hi shell for the estimation of the kinetic energy. Then, the age of the shell would be $t=0.3 R_{\mathrm{pc}} / v_{\exp }=$ $0.3 \mathrm{Myr}$ at $\mathrm{d}=1.5 \mathrm{kpc}$.

The total Hi mass and the kinetic energy of the Hi shell in the systemic or expansion velocity and distance domains are shown in Figure 14 (left and middle). The mass used for the estimation of the kinetic energy adopts a cosmic Helium abundance of $10 \%$. If G192 


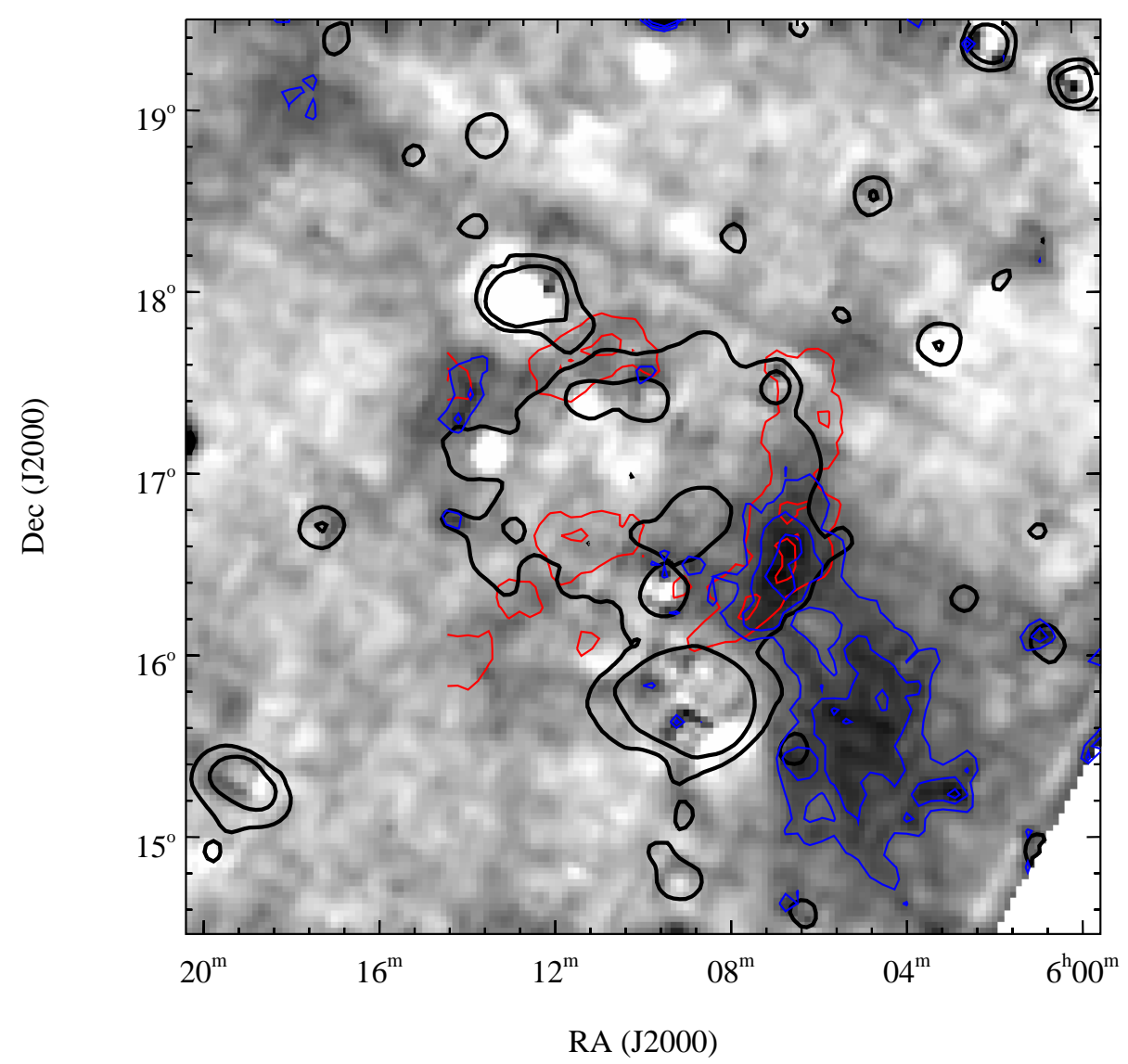

Figure 11. The red contours of the Hi emission indicating the Hi shell at $v_{\mathrm{LSR}}=+21 \mathrm{~km} \mathrm{~s}^{-1}\left(T_{b}=88\right.$ and $99 \mathrm{~K}$, see also Figure 4) are overlaid on the non-thermal radio continuum emission, of which the 3,4 , and $5 \sigma$ contours are presented with blue contours. The 0.2 and $0.6 \mathrm{~K}$ contours of the Effelsberg 21-cm radio continuum emission are presented in black to indicate the position of the G192 structure. The non-thermal radio continuum excess in the SE boundary of G192 appears to have a good spatial correlation with the HI shell at $v_{\mathrm{LSR}}=+21 \mathrm{~km} \mathrm{~s}^{-1}$.

is associated with the Gemini OB1 molecular clouds, i.e., $v_{\text {sys }}=+10 \mathrm{~km} \mathrm{~s}^{-1}$ and $\mathrm{d}=1.5 \mathrm{kpc}$, the Hi shell has $\mathrm{M}_{H I}=1.5 \times 10^{4} \mathrm{M}_{\odot}$ and $\mathrm{E}_{K}=0.08 \times 10^{51} \mathrm{erg}$. The physical parameters of the Hi shell estimated at a distance of $1.5 \mathrm{kpc}$ are summarized in Table 3 . If it is associated with $\mathrm{S} 259$ at $8.3 \mathrm{kpc}$ with $v_{\mathrm{sys}}=+20 \mathrm{~km} \mathrm{~s}^{-1}$, it has $\mathrm{M}_{H I}=2.0 \times 10^{5} \mathrm{M}_{\odot}$ and $\mathrm{E}_{K}=0.3 \times 10^{51} \mathrm{erg}$. We note that the total mass and the kinetic energy depend not only on distance but also on systemic velocity, because the velocity range where the mass distribution is flat shrinks or expands when the systemic velocity changes, as shown in Figure 8.

The initial expansion energy, $E_{\text {init }}$, which is defined by Heiles (1984) to be the equivalent energy that would have to be instantaneously deposited at the shell center to account for the current radius and expansion velocity of the shell, is higher than the observed kinetic energy, unless the energy loss due to radiative cooling is negligible. It is given by $E_{\text {init }}=5.3 \times 10^{43} n_{0}^{1.12} R^{3.12} v_{\text {exp }}^{1.4}$ ergs (Chevalier 1974) and it is often used to characterize Hi shell energetics (e.g., McClure-Griffiths et al. 2002). The estimated initial energy of the Hi shell at $d$ $=1.5 \mathrm{kpc}$ is $10^{51} \mathrm{erg}$, while it is 5 times higher at $\mathrm{d}=$
$8.3 \mathrm{kpc}$. The initial expansion energy in the parameter space of distance and expansion velocity is presented in Figure 14 (right).

The estimated initial energy of the shell at $1.5 \mathrm{kpc}$ happens to be the same as the canonical energy of an SN explosion. If the Hi shell is formed from stellar winds, the wind luminosity required to drive the Hi shell is $L_{W}=77 E_{K} /\left(9 R / v_{\text {exp }}\right)=2.2 \times 10^{37} \mathrm{erg} \mathrm{s}^{-1}$ (Weaver et al. 1977), when radiative losses are negligible. This corresponds to that of $\mathrm{O} 4 \mathrm{~V}, L_{\mathrm{O}} \mathrm{V}=2.9 \times 10^{37} \mathrm{erg}$ (Abbott 1982). Since radiative losses would not be negligible in case of G192, the required wind luminosity would be higher. A few early type stars (Reed 2005) are in the LOS of G192, but none appears to be responsible for the Hi shell. No stars seem to be near the center of G192, as shown in Figure 1. The stars in the field are no brighter than B0V, except the one associated with S261 (see Table 4), and the wind luminosity of B0V is three orders of magnitude smaller than that of the O4V star (Abbott 1982). Therefore, the known early type stars that are not associated with the known HiI regions cannot explain the $\mathrm{HI}_{\mathrm{I}}$ shell, while the explosion of an SN can. 


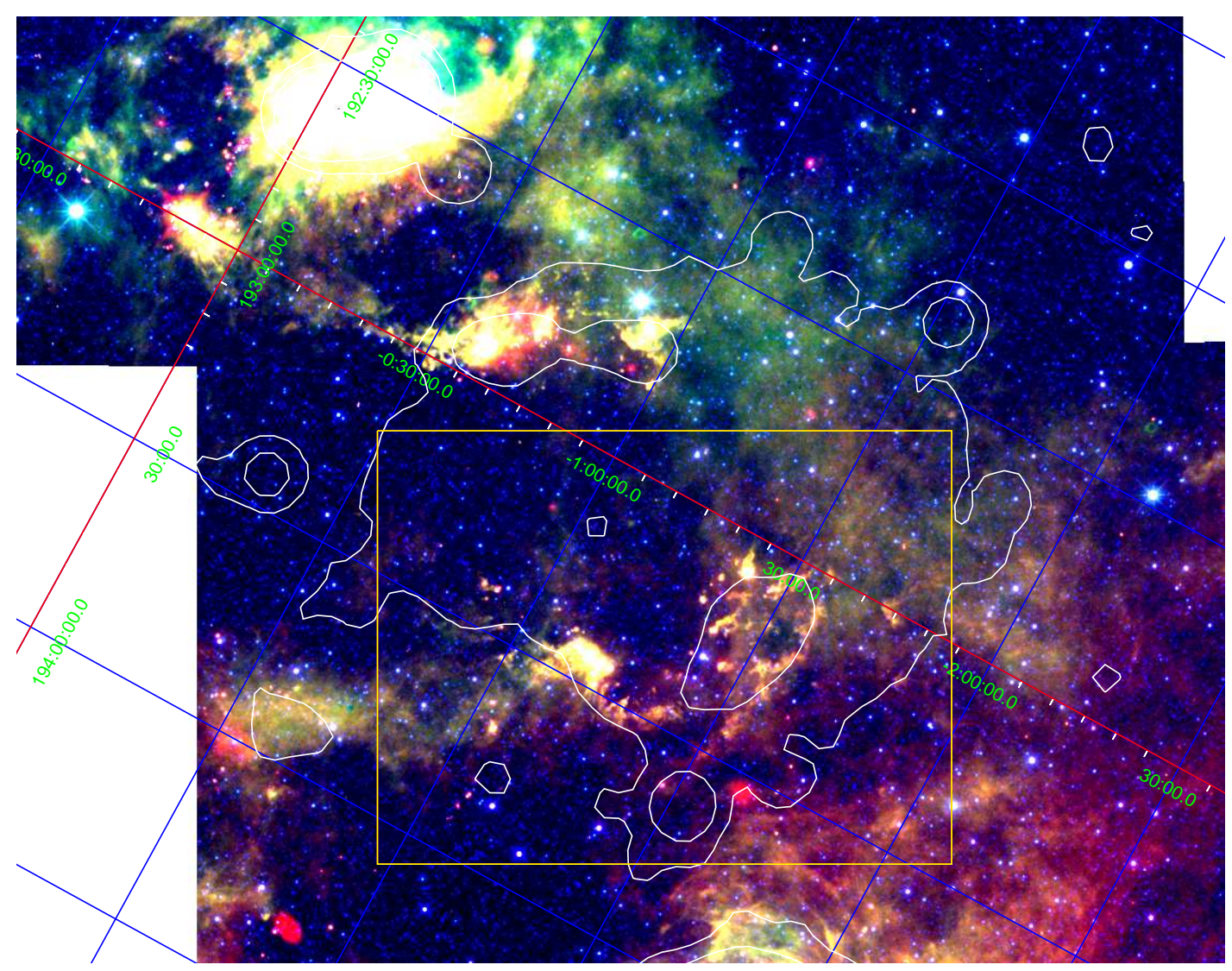

Figure 12. An RGB composite of the WISE 4.6 (blue), 12 (green), and $22 \mu \mathrm{m}$ (red) images of the G192 field. The 0.06 and $0.18 \mathrm{~K}$ contours of the 11-cm radio continuum data are overlaid. The yellow box shows the region of Figure 13 .

\subsection{Thermal Radio Continuum}

The radio continuum emission of the plateau and the southern concentration, which are considered to be associated to G192 originally, are observed to be thermal. We estimate the electron number density of the ionized medium and the required Lyman continuum photons, if it is photo-ionized. We also discuss possible sources for the thermal radio continuum.

The mean brightness temperature of the thermal plateau measured in the $11-\mathrm{cm}$ radio continuum image (Figure 1 ) is $T_{b}=0.08 \pm 0.02 \mathrm{~K}$. From this, we obtain the radio intensity of $I_{11 \mathrm{~cm}}=2 k T_{b} / \lambda^{2}=1.8 \times 10^{-19}$ erg $\mathrm{cm}^{-2} \mathrm{~s}^{-1} \mathrm{sr}^{-1} \mathrm{~Hz}^{-1}$, where $k$ is the Boltzmann constant and $\lambda$ is the wavelength. We assume that this is same as the intensity of ionized gas in the LOS described as free-free emissivity, $j_{f f, \nu}$, integrated over the length, $l$, which is given by

$$
\begin{aligned}
I_{\nu}= & \int j_{f f, \nu} d l=1.7 \times 10^{-22} g_{f f} \\
\times & \left(\frac{T}{10^{4} \mathrm{~K}}\right)^{-0.5} \frac{E M}{\mathrm{~cm}^{-6} \mathrm{pc}} \\
& \quad \operatorname{erg~cm} \mathrm{cm}^{-2} \mathrm{~s}^{-1} \mathrm{sr}^{-1} \mathrm{~Hz}^{-1}
\end{aligned}
$$

at radio frequencies, if the medium is assumed to be purely hydrogen. Here, $E M\left(\equiv \int n_{e}^{2} d l\right)$ is an emission measure. The Gaunt factor, $g_{f f}$, depends weakly on temperature and frequency, and $g_{f f}=5.5$ at $11-\mathrm{cm}$ in a medium with $T=10^{4} \mathrm{~K}$ (Equation 10.7 of Draine 2011). From Equation (5), the emission measure of the plateau, $E M=190 \mathrm{~cm}^{-6} \mathrm{pc}$, gives an electron number density of $n_{e}\left(\equiv \sqrt{\left\langle n_{e}^{2}\right\rangle}\right)=2.1 \mathrm{~cm}^{-3}$, when the line-of-sight depth of the medium is assumed to be the diameter of G192, i.e., 42 pc. The radio continuum concentration to the south with $T_{b}=0.12 \mathrm{~K}$ has $E M=$ $290 \mathrm{~cm}^{-6} \mathrm{pc}$ and $n_{e}=4.7 \mathrm{~cm}^{-3}$ when an angular size of $30^{\prime}$ is assumed. We note that the estimated EM can be 3 to 10 times larger, if the temperature of the medium is as hot as $10^{5}-10^{6} \mathrm{~K}$. If the ionized gas is clumped, $n_{e}=\sqrt{\left\langle n_{e}^{2}>\right.} f_{v}^{-1 / 6}$, where $f_{v}$ is the volume filling factor of the ionized gas.

If the continuum plateau is an HiI region photoionized by a star, we can calculate the required Lyman continuum photon luminosity, $Q$. The EM has a relation with $Q$, the hydrogen number density, $n_{H}$, and the temperature of the medium as the following.

$$
\begin{aligned}
E M & =4.22 \times 10^{4}\left(\frac{Q}{10^{49} \mathrm{~s}^{-1}}\right)^{1 / 3}\left(\frac{n_{H}}{10^{2} \mathrm{~cm}^{-3}}\right)^{4 / 3} \\
& \times\left(\frac{T}{10^{4} \mathrm{~K}}\right)^{0.28} \mathrm{~cm}^{-6} \mathrm{pc}
\end{aligned}
$$

when the rates of ionization and recombination are assumed to be in balance (Equation 15.4 of Draine 2011). 


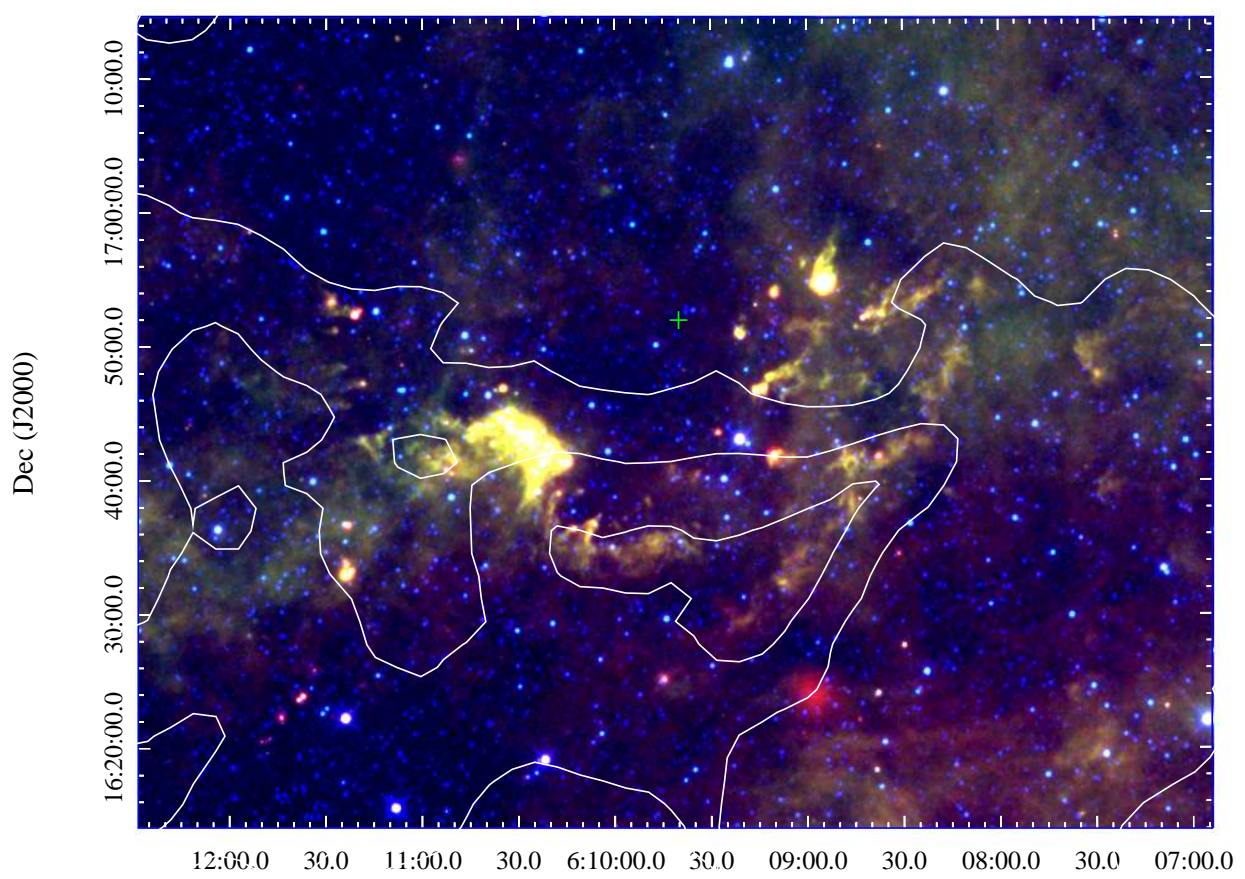

RA (J2000)

Figure 13. The magnified WISE image of the star forming region presented with a yellow box in Figure 12. Contours of the HI emission averaged over $v_{\mathrm{LSR}}=+27$ to $+32 \mathrm{~km} \mathrm{~s}^{-1}$ are overlaid. See the Hi channel image at $v_{\mathrm{LSR}}=+29.6 \mathrm{~km} \mathrm{~s}{ }^{-1}$ in Figure 4 for comparison. Contour levels are 23, 36, and $51 \mathrm{~K}$ in brightness temperature. The possible location of the central star traced by the direction of core-tail features in the eastern area of the bubble is indicated with a green cross symbol.

When $n_{H}=n_{e}$, we have $Q=4.6 \times 10^{48} \mathrm{~s}^{-1}$, which corresponds to that of a star with a spectral type of $\mathrm{O} 7 \mathrm{~V}$ - O7.5V (Martins et al. 2005). Since the HiI region disappears quickly after the central star leaves the main sequence, there must be an O7V star, or multiple stars that can produce that much amount of luminosity in total. The southern continuum concentration area has $Q=6.7 \times 10^{47} \mathrm{~s}^{-1}$, which corresponds to that of $09.5 \mathrm{~V}$. As shown in Table 4, no such stars are known in the central area of G192 or in the southern continuum concentration region.

We can think of a blister type HiI region, where a massive star makes an HiI region toward one direction, but the star is behind a molecular cloud and optically invisible. We searched IRAS point sources (Helou \& Walker 1988) in the LOS of G192, and investigated if their IR fluxes match those of massive stars. The IRAS point sources with detection ranks greater than 2 at 60 and $100 \mu \mathrm{m}$ are presented as triangles in Figure. 1. The bolometric luminosities of IRAS sources can be derived using the far infrared fluxes as follows (Lee et al. 1996; Kim \& Koo 2002).

$$
L_{I R}=0.39 R\left(\bar{T}_{d}, \beta\right)\left(F_{100}+2.58 F_{60}\right) d^{2} L \odot,
$$

where $R$ is of order 1 (Hildebrand 1983), and $F_{100}$ and $F_{60}$ are IRAS fluxes at 100 and $60 \mu \mathrm{m}$. Calculated luminosities of the brightest 5 sources are summarized in Table 5. According to Panagia (1973), $\log (L / L \odot)$ of B0 - B3 stars are $4.4-3$. The strongest in Table 5 has $\log (L / L \odot)=2.71$, which is smaller than that of
B3. Even at $\mathrm{d}=8.3 \mathrm{kpc}$, the listed 5 IRAS sources are no more than B0. Therefore, we conclude that there are no known stars in optical or in IR that could be responsible for the observed thermal radio continuum of G192.

\subsection{G192 as an Evolved Thermal-composite SNR Interacting With Nearby Massive Stars}

The estimated energy of the Hi shell and the possible association of the non-thermal radio continuum emission with the Hi shell suggest G192 to have an SN origin. However, the thermal radio continuum emission filling inside the Hi shell does not fit to the general evolutionary features of an old SNR. The morphology of the thermal radio continuum and the surrounding Hi shell have an excellent correlation. Thus, it does not seem to be a chance coincidence. We discuss possible scenarios to explain these conflicting observational results.

First, we examine the possibility that the thermal radio continuum arises from the hot gas of $T=10^{6-7} \mathrm{~K}$ residing inside an SNR. G192 is not detected in any of the 6 bands in the diffuse background ROSAT all sky data (Snowden et al. 1997). The expected count rate per $E M$ is $8 \times 10^{-4}$ counts s$^{-1} \operatorname{arcmin}^{-2}$ at $T=$ $5 \times 10^{6} \mathrm{~K}$ in band $5(0.56-1.21 \mathrm{keV})$, for example, when a maximum extinction of $N_{H} \sim 10^{22} \mathrm{~cm}^{-2}$ is assumed (see Figure 7 of Snowden et al. 1997). The count rate of $\mathrm{G} 192$, for an $E M=190 \mathrm{~cm}^{-6} \mathrm{pc}$, would be 0.16 counts s$^{-1}$ arcmin $^{-2}$, while, the rms of the G192 

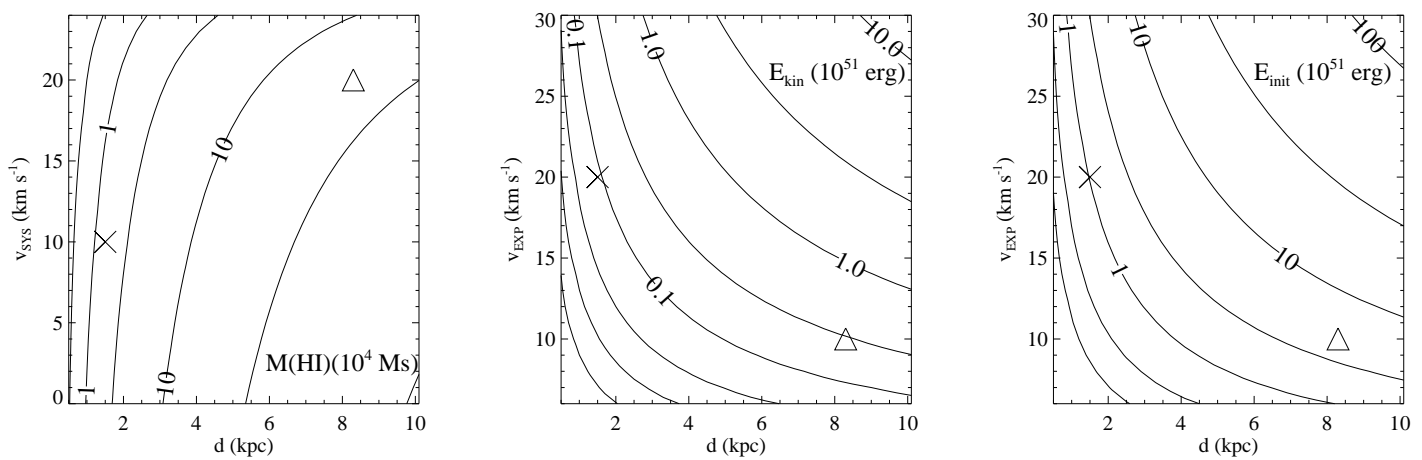

Figure 14. Total Hi mass (left), kinetic (middle), and initial energy (right) of the Hi shell as function of distance and systemic, or expansion velocities in units of $10^{4} \mathrm{M}_{\odot}$ and $10^{51} \mathrm{erg}$. The cross and triangle symbols indicate the locations of the shell parameters at the distances of the Perseus and Outer Arms, respectively.

Table 1

Sharpless HiI regions in the observed field

\begin{tabular}{ccrrrr}
\hline Name & $\begin{array}{c}\text { RA, Dec (J2000) } \\
\left(\mathrm{h} \mathrm{m},{ }^{\circ}\right)\end{array}$ & $\begin{array}{r}\text { Diameter }^{\mathrm{a}} \\
\left({ }^{\prime}\right)\end{array}$ & $\begin{array}{r}v_{\mathrm{LSR}}{ }^{\mathrm{b}} \\
\left(\mathrm{km} \mathrm{s}^{-1}\right)\end{array}$ & $\begin{array}{r}v_{\mathrm{LSR}^{\mathrm{c}}} \\
\left(\mathrm{km} \mathrm{s}^{-1}\right)\end{array}$ & $\begin{array}{r}\text { Distance }^{\mathrm{d}} \\
(\mathrm{kpc})\end{array}$ \\
\hline $\mathrm{S} 254$ & $0612.3,+1803$ & 11 & +11.1 & +7.5 & 1.6 \\
$\mathrm{~S} 255$ & $0613.1,+1759$ & 3 & +6.7 & +7.5 & 1.6 \\
$\mathrm{~S} 256$ & $0612.6,+1757$ & 1 & +6.0 & +7.5 & 1.6 \\
$\mathrm{~S} 257$ & $0612.8,+1759$ & 3 & +11.0 & +7.5 & 1.6 \\
$\mathrm{~S} 258$ & $0613.5,+1755$ & 1 & +7.9 & +8.0 & 1.6 \\
$\mathrm{~S} 259$ & $0611.6,+1727$ & 2 & $-23.0,+15.8$ & +22.8 & 8.3 \\
$\mathrm{~S} 261$ & $0608.9,+1548$ & 45 & +5.6 & $\ldots$ & 1.4 \\
\hline
\end{tabular}

a Optical size (Sharpless 1959)

b H $\alpha$ emission line radial velocity (Fich et al. 1990), except S261, which is from Hausen et al. (2002).

c CO radial velocity (Blitz et al. 1982). For S261, the association is inconclusive.

d Distances determined by spectrophotometry of the central ionizing stars by Moffat et al. (1979) for S259 and by Georgelin \& Georgelin (1976) for S261. Distance of S255 is measured by parallaxes using the methanol maser (Rygl et al. 2010), and the distances for S254 S258 are assumed to be the same as the S255

region is only $\sim 10^{-4}$ counts $\mathrm{s}^{-1} \operatorname{arcmin}^{-2}$, meaning that it should have been detected if the gas is at such high temperatures. Therefore, the thermal electrons emitting in the radio cannot be that hot.

Considering the low shock speed and the age of G192, we suspect that the hot gas originally inside the SNR might have cooled down. To check this, we first consider the condition of the SNR at the time of shell formation by following the analysis of Cox et al. (1999). The radiative shell formation time of an SNR embedded in a medium with an ambient gas density of $n_{0}=16 \mathrm{~cm}^{-3}$ and with an explosion energy of $E_{51} \equiv E /\left(10^{51} \mathrm{erg}\right)=1$ is $t_{s h}=5.3 \times 10^{4} E_{51}^{3 / 14} / n_{0}^{4 / 7}=$ $11,000 \mathrm{yr}$. At this moment, the radius of the remnant is $R_{s h}=12.5 E_{51}^{0.2} n_{0}^{-0.2} t_{4}^{0.4}=7.5$ pc where $t_{4} \equiv t / 10^{4}$ yrs, and the shock speed and the post-shock temperature are $v_{s h}=490 E_{51}^{0.2} n^{-0.2} t_{4}^{-0.6}=270 \mathrm{~km} \mathrm{~s}^{-1}$ and $T_{s h}=$ $1.38 \times 10^{5} v_{s h}^{2}=1.0 \times 10^{6} \mathrm{~K}$, respectively. In the presence of unsuppressed thermal conduction, the density of the central hot gas at the time of the shell formation is about $0.13 n_{o} \approx 2.1 \mathrm{~cm}^{-3}$ (Cox et al. 1999), and the central temperature could be close to $\sim 5 \times 10^{6} \mathrm{~K}$ (Figure 11 of Shelton et al. 1999). Then, the condition of G192 at the time of shell formation would be

$$
n=2.1 \mathrm{~cm}^{-3}, T \sim 5 \times 10^{6} \mathrm{~K}: \text { central region }
$$

$$
n=4 n_{0}=64 \mathrm{~cm}^{-3}, T \sim 1 \times 10^{6} \mathrm{~K}: \text { boundary } .
$$

This hot gas will cool down as the SNR expands by radiation, expansion, and thermal conduction (e.g., Kawasaki et al. 2002). If we use the result of Cioffi et al. (1988, Equation 3.16b), which accounts for both adiabatic and radiative cooling effects, the interior temperature drops as $T \propto R^{-3.5}$. Then, the temperature of the hot gas at the time of shell formation would decrease down to $1.3 \times 10^{5} \mathrm{~K}$, when the shell reaches to the current radius of G192, $21 \mathrm{pc}$. The radiative cooling time of such gas is given by

$$
\tau_{\text {cool }}=\frac{3 n k T}{2 \Lambda}=2,200\left(\frac{T}{10^{5} \mathrm{~K}}\right)^{1.7}\left(\frac{1 \mathrm{~cm}^{-3}}{n_{H}}\right) \mathrm{yrs}
$$

where the volume of the gas is assumed to be constant (Equation 34.4 of Draine 2011). Here, $n$ is the number density of free particles, and the cooling function of Dere et al. (2009), $\Lambda / n_{e} n_{H} \sim 1.1 \times$ $10^{-22}\left(T / 10^{6} K\right)^{-0.7}$ erg $\mathrm{cm}^{3} \mathrm{~s}^{-1}$, for a temperature range of $10^{5}<T<10^{7.3} \mathrm{~K}$ is adopted. This equation means that the probability for the gas to have temperatures $T \sim 100,000-10,000 \mathrm{~K}$ in the process of cooling is very low, since it would then rapidly cool down to the temperature of the ambient medium $(<2000 \mathrm{yrs})$ 
Table 2

Spectral indices of thermal and non-thermal regions

\begin{tabular}{lllll}
\hline Method & SW & NE & Plateau & S261 \\
\hline$\alpha$ Map Median & $0.57 \pm 0.05$ & $0.85 \pm 0.32$ & $0.06 \pm 0.07$ & $0.05 \pm 0.05$ \\
Total Flux & $0.55 \pm 0.15$ & $0.83 \pm 0.35$ & $0.04 \pm 0.07$ & $0.04 \pm 0.03$ \\
T-T Plot & $0.40 \pm 0.15$ & $0.31 \pm 0.23$ & $0.11 \pm 0.01$ & $0.05 \pm 0.01$ \\
\hline
\end{tabular}

Table 3

Parameters of the Hi expanding shell

\begin{tabular}{ll}
\hline Parameter & Estimated value \\
\hline Center $(\alpha, \delta)$ & $06^{\mathrm{h}} 09^{\mathrm{m}} 04^{\mathrm{s}},+17^{\circ} 05^{\prime}$ \\
Angular radius & $0^{\circ} .8$ \\
Mean physical radius & $21 d_{1.5 \mathrm{kpc}} \mathrm{pc}$ \\
Mean expansion velocity & $20 \mathrm{~km} \mathrm{~s}^{-1}$ \\
Total Hi mass & $1.5 \pm 0.4 \times 10^{4} d_{1.5 \mathrm{kpc}}^{2} \mathrm{M}_{\odot}$ \\
Kinetic energy $(\mathrm{H}+\mathrm{He})$ & $0.08 \pm 0.02 \times 10^{51} d_{1.5 \mathrm{kpc}}^{2} \mathrm{erg}$ \\
Initial ISM density & $16 \pm 4 d_{1.5 \mathrm{kpc}}^{-1} \mathrm{~cm}^{-3}$ \\
Age & $0.32 d_{1.5 \mathrm{kpc}} \mathrm{Myr}$ \\
Distance & $1.5 \mathrm{kpc}$ \\
\hline
\end{tabular}

If there are dense swept-up cloudlets inside hot gas, the cooling time would be even shorter (e.g., White \& Long 1991). Therefore, the hot gas originally inside the SNR should be in neutral state by now, therefore not emitting in radio frequencies, unless there is (an) external source(s) re-ionizing the neutralized gas.

The radio continuum surface brightness distribution of G192 is interesting in the sense that it appears brighter near the center and becomes weaker where the density of the Hi shell peaks. It is different from that of a typical shell-type SNR, where the gas density dramatically decreases towards the center. The estimated electron number density of $n_{e} \sim 2 \mathrm{~cm}^{-3}$ is also larger than that of a typical shell-type SNR (e.g., Slavin \& Cox 1992). Note also that the estimated electron number density is too small in comparision to that of the evolved Hir regions. These suggest that G192 used to be one of the so called "Thermalcomposite" (Seward 1990; Jones et al. 1998) or "Mixedmorphology" SNRs (Rho \& Petre 1998), which are SNRs with center-filled thermal X-ray emission surrounded by non-thermal radio continuum shell. Physical processes of thermal composite SNRs can be found in Vink (2012) and Kawasaki et al. (2005). The total gas mass confined within the Hi shell estimated from the radio continuum of G192 is about $450 \mathrm{M}_{\odot}$. Considering the uncertainties of measured electron number density, this is not very different from those of known thermal composite SNRs (e.g., Lazendic \& Slane 2006). In addition, if we apply the same analysis above to W44, a 0.02 Myr-old thermal composite SNR with $R=12 \mathrm{pc}$, we obtain a similar result that the hot central gas of W44 with $T_{c}=6 \times 10^{6} \mathrm{~K}$, and $n_{c} \sim 1 \mathrm{~cm}^{-3}$ (Cox et al. 1999) would also cool down, when it is as old as G192. Therefore, it appears to be reasonable to consider G192 as an evolved form of W44, i.e., a cooled thermal composite SNR.
To reignite the radio continuum emission of the neutralized gas inside G192, external ionizing sources are required. Since massive stars tend to be born in groups in molecular clouds, it is possible that an evolved SNR encounters a nearby massive main sequence star. The WISE images of Figure 12 and Figure 13 reveal hints of the putative ionized star. The infrared counterpart of the southern continuum concentration appears as an U-shaped star forming region opened to the north, and it shows excellent correlation with the Hi shell, meaning they are associated. The morphology of the Ushaped region implies the presence of a central ionizing star, somewhere around $(\alpha, \delta) \sim\left(6^{\mathrm{h}} 09^{\mathrm{m}} 40^{\mathrm{s}},+16^{\circ} 52^{\prime}\right)$, which is offset from the center and close to the northern boundary of the U-shaped structure. Since it is opened towards the low density area $\left(n_{H} \sim 2 \mathrm{~cm}^{-3}\right)$, the stellar photons can easily spread out to the diffuse interior of G192, re-ionizing the cooled neutral gas left inside the SNR. The existence of an O7V star or multiple stars with comparable Lyman photons near the southern concentration can fulfill the presence of the centrally brightened thermal emission together with the Hi shell and the non-thermal continuum emission.

\section{Conclusion}

G192 has been considered to be an SNR, but recently the radio continuum emission has been proven to be thermal. By comparing the $\mathrm{HI}$, the radio continuum, and the infrared emission, we propose G192 to be an aged "thermal composite" SNR brightened again in thermal radio continuum by one or more nearby massive stars. Our main findings are summarized as follows.

1. We have re-examined the radio continuum emission of the G192 field using the 21 -and the 6 -cm radio continuum data. We have confirmed that the central plateau and the southern concentration, which comprise the traditional G192, have flat thermal spectra of $\alpha \sim 0$. The emission measure and the electron density of the thermal radio continuum plateau are $E M$ $=190 \mathrm{~cm}^{-6} \mathrm{pc}$ and $n_{e}=2.1 \mathrm{~cm}^{-3}$, which requires a Lyman photon number rate corresponding to that of a massive star with a spectral type of O7V - O7.5V. We have searched possible progenitors of the thermal radio continuum emission, but found no stars in optical and infrared that could be responsible for the observed thermal emission. Importantly, we have revealed the existence of faint non-thermal radio continuum emission with $\alpha \sim 0.56$ in the southwestern area of G192, which lies outside the thermal continuum plateau.

2. We have performed high-resolution Hi $21 \mathrm{~cm}$ line 
Table 4

Early type stars in the G192 field

\begin{tabular}{lclcc}
\hline Name & $\begin{array}{c}\text { RA, Dec }(\mathrm{J} 2000) \\
\left.\left(\mathrm{h} \mathrm{m},{ }^{\circ}\right)^{\prime}\right)\end{array}$ & Spectral Type & $\begin{array}{c}\text { Distance } \\
(\mathrm{kpc})\end{array}$ & $\begin{array}{c}\text { Associated } \\
\text { His Region }\end{array}$ \\
\hline HD 41997 & $0608.9,+1542$ & O7 & 1.4 & $\mathrm{~S} 261$ \\
HD 42476 & $0611.7,+1723$ & B9.5III & 8.3 & S259 \\
HD 253250 & $0612.2,+1535$ & B9II & $\ldots$ & $\ldots$ \\
HD 253247 & $0612.4,+1801$ & B1V & 1.6 & S254 \\
HD 253327 & $0612.7,+1759$ & B0V & 1.6 & S257 \\
LS 19 & $0613.1,+1759$ & B0V & 1.6 & S255 \\
HD 253659 & $0613.9,+1631$ & B0.5V & $\ldots$ & $\ldots$ \\
HD 253658 & $0613.9,+1711$ & B8Ib & $\ldots$ & $\ldots$ \\
HD 43496 & $0617.1,+1551$ & B8III & $\ldots$ & $\ldots$ \\
\hline
\end{tabular}

The early-type stars in the area of G192 (Reed 2005). See also Figure 1. The references for the distances are the same as in Table 1.

Table 5

Luminosities of strong IRAS point sources in the G192 field

\begin{tabular}{lccc}
\hline & RA, Dec (J2000) & & \\
Name & $\left(\mathrm{h} \mathrm{m},{ }^{\circ}{ }^{\prime}\right)$ & $\log \left(L_{1.5 \mathrm{kpc}} / \mathrm{L} \odot\right)$ & $\log \left(L_{8.3 \mathrm{kpc}} / \mathrm{L} \odot\right)$ \\
\hline IRAS 06084+1727 & $61124.5,+172627$ & 2.71 & 4.20 \\
IRAS 06075+1643 & $61027.4,+164318$ & 2.32 & 3.81 \\
IRAS 06060+1655 & $60854.9,+165436$ & 2.15 & 3.64 \\
IRAS 06069+1729 & $60950.1,+172835$ & 2.05 & 3.53 \\
IRAS 06089+1727 & $61152.5,+172645$ & 1.53 & 3.02 \\
\hline
\end{tabular}

The bolometric luminosities of the top five brightest IRAS point sources in the field of G192 when they are assumed to be at a distance of $1.5 \mathrm{kpc}$ and $8.3 \mathrm{kpc}\left(L_{1.5 \mathrm{kpc}}\right.$ and $\left.L_{8.3 \mathrm{kpc}}\right)$ are presented in units of the solar luminosities.

observations toward G192 using the Arecibo $305 \mathrm{~m}$ radio telescope. We have detected receding parts of an expanding Hi shell around the thermal radio continuum. The shell is very thick $(\sim 40 \%$ of radius $)$ and parts of the shell expand with up to $v_{\text {exp }}=50-60 \mathrm{~km}$ $\mathrm{s}^{-1}$. We assume G192 to be associated with the Gemini OB1 molecular clouds at $d=1.5 \mathrm{kpc}$. By fitting the mass distribution of the Hi shell, we have estimated a radius of $21 \mathrm{pc}$, an initial ISM density of $16 \mathrm{~cm}^{-3}$, a total Hi mass of $1.5 \times 10^{4} \mathrm{M}_{\odot}$, and a characteristic expansion velocity of $\sim 20 \mathrm{~km} \mathrm{~s}^{-1}$. The initial expansion energy of the shell is $1 \times 10^{51} \mathrm{erg}$ at $\mathrm{d}=1.5 \mathrm{kpc}$, which corresponds to the canonical explosion energy of an SN.

3. We have compared the thermal and non-thermal radio continuum emission with the $\mathrm{HI}$ shell and have found a tight spatial correlation between the Hi shell and the thermal emission, and some correlation between the Hi shell and the non-thermal emission. We have also compared these features with the WISE highresolution infrared image. We have found an excellent correlation between the Hi shell and the infrared counterparts of the southern continuum rim, which appears to be a star forming infrared bubble. The good morphological correlations between multiple wavelengths imply their physical association.

4. The estimated parameters of the Hi shell and the possible association of the non-thermal continuum emission with the Hi shell suggest G192 being an SNR. However, the centrally-peaked thermal radio emission is not consistent with the standard SNR model. We argue that G192 was a thermal composite SNR with a more-or-less uniformly filled hot gas, which later cooled down and re-ionized by one or more massive stars. The infrared image supports that the southern area of G192 is currently encountering a nearby star forming region that possibly contains possible multiple massive stars.

\section{ACKNOWLEDGMENTS}

We are grateful to Kee-Tae Kim, Jae-Joon Lee, and Kwang-Il Sun for their suggestions and comments. J.-h. K. and B.-D. Y. acknowledge support from KASI-Yonsei DRC program of Korea Research Council of Fundamental Science and Technology(DRC-12-2KASI). B,-C. K. has been supported by the Korean Research Foundation under grant KRF-2008-313-C00372. The Arecibo Observatory is operated by SRI International under a cooperative agreement with the National Science Foundation (AST-1100968), and in alliance with Ana G. Mndez-Universidad Metropolitana, and the Universities Space Research Association.

\section{REFERENCES}

Abbott, D. C. 1982, The Return of Mass and Energy to the Interstellar Medium by Winds from Early-Type Stars, ApJ, 263, 723

Arendt, R. G. 1989, An Infrared Survey of Galactic Supernova Remnants, ApJS, 70, 181

Berkhuijsen, E. M. 1974, The Origem Loop - an Old Supernova Remnant in the Anticentre, A\&A, 35, 429

Blitz, L., Fich, M., \& Stark, A. A. 1982, Catalog of CO 
Radial Velocities toward Galactic H II Regions, ApJS, 49, 183

Brand, J., \& Blitz, L. 1993, The Velocity Field of the Outer Galaxy, A\&A, 275, 67

Carpenter, J. M., Snell, R. L., \& Schloerb, F. P. 1995, Anatomy of the Gemini OB1 Molecular Cloud Complex, ApJ, 445, 246

Caswell, J. L. 1970, Radio Observations of Four Supernova Remnants, Australian Journal of Physics, 23, 105

Caswell, J. L. 1985, Is G192.8-1.1 a Discrete Supernova Remnant, or Part of the Origem Loop?, AJ, 90, 1076

Cazzolato, F., \& Pineault, S. 2005, Theoretical and Observational Aspects of Expanding HI Shells, AJ, 129, 2731

Chevalier, R. A. 1974, The Evolution of Supernova Remnants: Spherically Symmetric Models, ApJ, 188, 501

Cioffi, D. F., McKee, C. F., \& Bertschinger, E. 1988, Dynamics of Radiative Supernova Remnants, ApJ, 334, 252

Condon, J. J., Cotton, W. D., Greisen, E. W., et al. 1998, The NRAO VLA Sky Survey, AJ, 115, 1693

Cox, D. P., Shelton, R. L., Maciejewski, W., et al. 1999, Modeling W44 as a Supernova Remnant in a Density Gradient with a Partially Formed Dense Shell and Thermal Conduction in the Hot Interior. I. The Analytical Model, ApJ, 524, 179

Day, G. A., Caswell, J. L., \& Cooke, D. J. 1972, A LowLatitude Galactic Survey from $\mathrm{l}=46$ to $61^{\circ}$ and 190 to $290^{\circ}$ at $2700 \mathrm{MHz}$, Australian Journal of Physics, 25, 1

Dere, K. P., Landi, E., Young, P. R., et al. 2009, CHIANTI - an Atomic Database for Emission Lines. IX. Ionization Rates, Recombination Rates, Ionization Equilibria for the Elements Hydrogen through Zinc and Updated Atomic Data, A\&A, 498, 915

Dickel, J. R., \& Denoyer, L. K. 1975, Low-Frequency Radio Maps and Spectra of Supernova Remnants, AJ, 80, 437

Draine, B. T. 2011, Physics of the Interstellar and Intergalactic Medium (Princeton: Princeton University Press)

Fich, M., Dahl, G. P., \& Treffers, R. R. 1990, Fabry-Perot H-Alpha observations of Galactic HII Regions, AJ, 99, 622

Fürst, E., Reich, W., Reich, P., \& Reif, K. 1990, A Radio Continuum Survey of the Galactic Plane at 11-cm Wavelength - Part Three - the Area $76<L<240^{\circ}$, A\&AS, 85,691

Fürst, E., Reich, W., Reich, P., \& Reif, K. 1990, A Radio Continuum Survey of the Galactic Plane at 11-cm Wavelength - Part Four - a List of 6483 Small Diameter Sources, A\&AS, 85, 805

Gao, X. Y., Han, J. L., Reich, W., et al. 2011, A SinoGerman 6-cm Polarization Survey of the Galactic Plane. V. Large Supernova Remnants, A\&A, 529, A159

Gao, X. Y., Reich, W., Han, J. L., et al. 2010, A SinoGerman 6-cm Polarization Survey of the Galactic Plane. II. The Region from 129 to $230^{\circ}$ Longitude, A\&A, 515, A64

Georgelin, Y. M., \& Georgelin, Y. P. 1976, The Spiral Structure of Our Galaxy Determined from HII Regions, A\&A, 49,57

Garmany, C. D., Conti, P. S., \& Chiosi, C. 1982, The Initial Mass Function for Massive Stars, ApJ, 263, 777

Giacani, E., \& Dubner, G. 2004, The Neutral Gas in the Environs of the Wolf Rayet Stars in the Circinus OB1 Association, A\&A, 413, 225

Green, D. A. 2009, A Catalogue of Galactic Supernova Remnants (2009 March version), Astrophysics Group, Cavendish Laboratory, Cambridge, United Kingdom
Hartmann, D., \& Burton, W. B. 1997, Atlas of Galactic Neutral Hydrogen (Cambridge: Cambridge Press)

Hausen, N. R., Reynolds, R. J., \& Haffner, L. M. 2002, Measurements of [O I] $\lambda 6300 / \mathrm{H} \alpha$ Line Intensity Ratios for Four O Star H II Regions, AJ, 124, 3336

Heiles, C. 1984, HI Shells, Supershells, Shell-Like Objects, and Worm, ApJS, 55, 585

Helou, G., \& Walker, D. W. 1988, Infrared Astronomical Satellite (IRAS) Catalogs and Atlases. Volume 7, p.1-265

Hildebrand, R. H. 1983, The Determination of Cloud Masses and Dust Characteristics from Submillimetre Thermal Emission, QJRAS, 24, 267

Jones, T. W., Rudnick, L., Jun, B.-I., et al. 1998, $10^{51}$ Ergs: The Evolution of Shell Supernova Remnants, PASP, 110, 125

Kang, J.-h., Koo, B.-C., \& Salter, C. 2012, An Old Supernova Remnant within an HII Complex : FVW 172.8+1.5, AJ, 143, 75

Kang, J.-h., \& Koo, B.-C. 2007, Faint H I 21-cm Emission Line Wings at Forbidden Velocities, ApJS, 173, 85

Kawasaki, M. T., Ozaki, M., Nagase, F., et al. 2002, ASCA Observations of the Supernova Remnant IC 443: Thermal Structure and Detection of Overionized Plasma, ApJ, 572,897

Kawasaki, M., Ozaki, M., Nagase, F., Inoue, H., \& Petre, R. 2005, Ionization States and Plasma Structures of MixedMorphology Supernova Remnants Observed with ASCA, ApJ, 631, 935

Kim, K.-T., \& Koo, B.-C. 2002, Interaction between Ionized and Molecular Gas in the Active Star-Forming Region W31, ApJ, 575, 327

Koo, B.-C., \& Heiles, C. 1991, A Survey of HI 21 Centimeter Emission Lines toward Supernova Remnants, ApJ, 382,204

Kothes, R., \& Kerton, C. R. 2002, Expanding Shells of Shocked Neutral Hydrogen around Compact H II Regions, A\&A, 390, 337

Lazendic, J. S., \& Slane, P. O. 2006, Enhanced Abundances in Three Large-Diameter Mixed-Morphology Supernova Remnants, ApJ, 647, 350

Lee, J.-J., Koo, B.-C., Yun, M. S., et al. 2008, A 21-cm Spectral and Continuum Study of IC 443 Using the Very Large Array and the Arecibo Telescope, AJ, 135, 796 A

Lee, Y., Snell, R. L., \& Dickman, R. L. 1996, The Cold, Massive Molecular Cloud G216-2.5. III. Infrared Properties, ApJ, 472, 275

Martins, F., Schaerer, D., \& Hillier, D. J. 2005, A New Calibration of Stellar Parameters of Galactic O Stars, A\&A, 436, 1049

McClure-Griffiths, N. M., Dickey, J. M., Gaensler, B. M., \& Green, A. J. 2002, The Galactic Distribution of Large H I Shells, ApJ, 578, 176

Milne, D. K. 1969, 6-cm Observations of Nonthermal Radio Sources Near the Galactic Plane, Australian Journal of Physics, 22, 613

Milne, D. K., \& Dickel, J. R. 1974, 2700 MHz Polarization Observations of 17 Supernova Remnants, Australian Journal of Physics, 27, 549

Moffat, A. F. J., Jackson, P. D., \& Fitzgerald, M. P. 1979, The Rotation and Structure of the Galaxy beyond the Solar Circle, A\&AS, 38, 197

Neugebauer, G., Habing, H. J., van Duinen, R., et al. 1984, The Infrared Astronomical Satellite (IRAS) Mission, ApJL, 278, L1

Panagia, N. 1973, Some Physical Parameters of Early-Type 
Stars, AJ, 78, 929

Park, G., Koo, B.-C., Gibson, S. J., et al. 2013, H I Shells and Supershells in the I-GALFA H I 21-cm Line Survey. I. Fast-Expanding H I Shells Associated with Supernova Remnants, ApJ, 777, 14

Reed, C. 2005, VizieR Online Data Catalog: Photometry and Spectroscopy for Luminous Stars, 5125

Reich, P., Reich, W., \& Furst, E. 1997, The Effelsberg 21 CM Radio Continuum Survey of the Galactic Plane between $\mathrm{L}=95.5 \mathrm{deg}$ and $\mathrm{L}=240$ deg., A\&AS, 126, 413

Rho, J., \& Petre, R. 1998, Mixed-Morphology Supernova Remnants, ApJL, 503, L167

Russeil, D., Adami, C., \& Georgelin, Y. M. 2007, Revised Distances of Northern HII Regions, A\&A, 470, 161

Rygl, K. L. J., Brunthaler, A., Reid, M. J., et al. 2010, Trigonometric Parallaxes of $6.7 \mathrm{GHz}$ Methanol Masers, A\&A, 511, A2

Saken, J. M., Fesen, R. A., \& Shull, J. M. 1992, An IRAS Survey of Galactic Supernova Remnants, ApJS, 81, 715

Seward, F. D. 1990, Einstein Observations of Galactic Supernova Remnants, ApJS, 73, 781

Sharpless, S. 1959, A Catalogue of H II Regions, ApJS, 4, 257

Shelton, R. L., Cox, D. P., Maciejewski, W., et al. 1999,
Modeling W44 as a Supernova Remnant in a Density Gradient with a Partially Formed Dense Shell and Thermal Conduction in the Hot Interior. II. The Hydrodynamic Models, ApJ, 524, 192

Slavin, J. D., \& Cox, D. P. 1992, Completing the Evolution of Supernova Remnants and Their Bubbles, ApJ, 392, 131

Snowden, S. L., Egger, R., Freyberg, M. J., et al. 1997, ROSAT Survey Diffuse X-Ray Background Maps II, ApJ, 485,125

Tamanaha, C. M. 1997, The Anticenter Shell and the Anticenter Chain, ApJS, 109, 139

van der Werf, P. P., \& Higgs, L. A. 1990, Radio and Infrared Observations of the H II Complex BG 2107+49, A\&A, 235,407

Vink, J. 2012, Supernova Remnants: the X-Ray Perspective, A\&A Rev., 20, 49

Weaver, R., McCray, R., Castor, J., Shapiro, P., \& Moore, R. 1977, Interstellar Bubbles. II - Structure and Evolution, ApJ, 218, 377

White, R. L., \& Long, K. S. 1991, Supernova Remnant Evolution in an Interstellar Medium with Evaporating Clouds, ApJ, 373, 543 